\title{
Characterization of Novel Small-Molecule NRF2 Activators: Structural and Biochemical Validation of Stereospecific KEAP1 Binding
}

Carlos Huerta $^{\mathrm{a}, \mathrm{b}}$, Xin Jiang ${ }^{\mathrm{c}}$, Isaac Trevino ${ }^{\mathrm{a}}$, Christopher F. Bender ${ }^{\mathrm{c}}$, Deborah A. Ferguson ${ }^{\mathrm{a}}$, Brandon

Probst $^{\mathrm{a}}$, Kerren K. Swinger ${ }^{\mathrm{d}, 1}$, Vincent S. Stoll ${ }^{\mathrm{d}}$, Philip J. Thomas ${ }^{\mathrm{b}}$, Irina Dulubova ${ }^{\mathrm{a}}$, Melean Visnick ${ }^{\mathrm{c}}$ and W. Christian Wigley ${ }^{\mathrm{a}}$

${ }^{\mathrm{a}}$ Department of Research, Reata Pharmaceuticals, Inc., Irving, TX 75063

${ }^{\mathrm{b}}$ Department of Physiology, UT Southwestern Medical Center, Dallas, TX 75390

${ }^{c}$ Department of Discovery Chemistry, Reata Pharmaceuticals, Inc., Irving, TX 75063

${ }^{\mathrm{d}}$ AbbVie Inc., North Chicago, IL 60064

Corresponding authors:

Melean Visnick, PhD; Telephone: (469) 442-4724; Email: mike.visnick@ reatapharma.com

W. Christian Wigley, PhD; Telephone: (972) 865-2209; Email: chris.wigley@ reatapharma.com

${ }^{1}$ Current address: Ribon Therapeutics, Inc., Lexington, MA, 02421 


\section{Graphical Abstract}
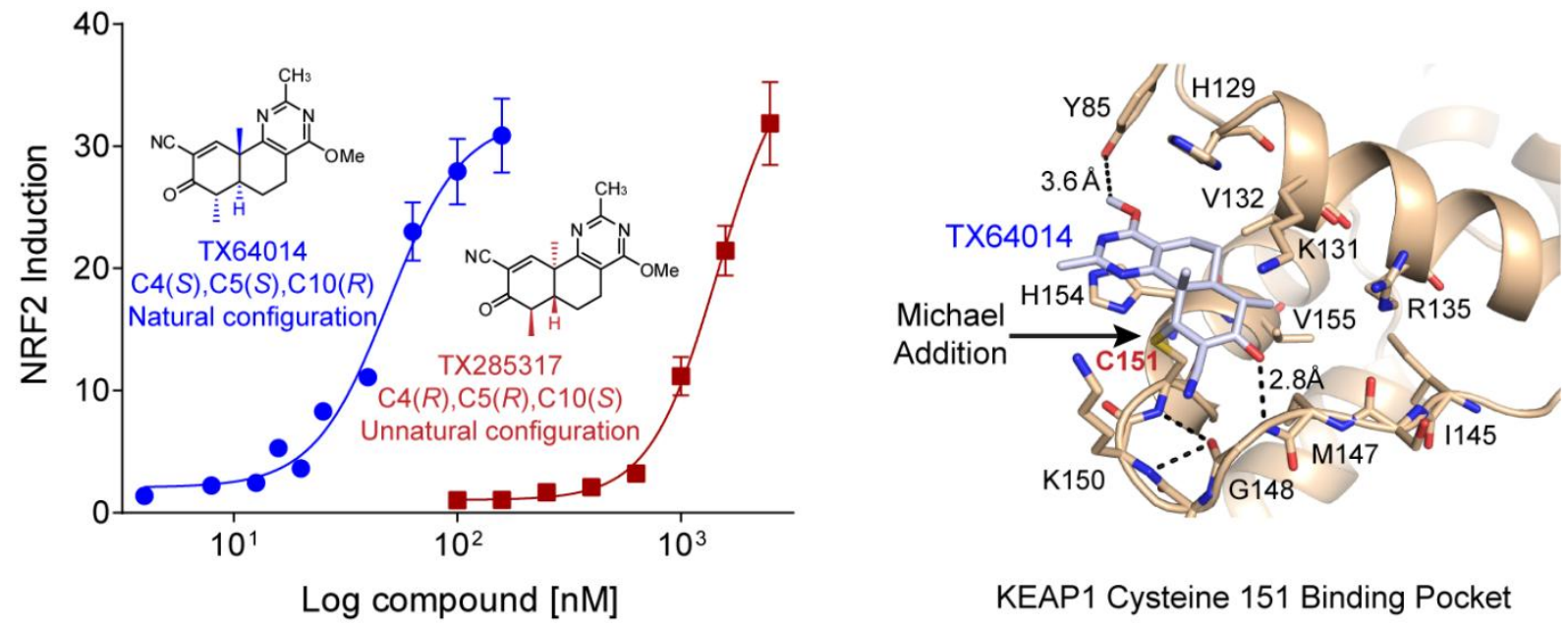

KEAP1 Cysteine 151 Binding Pocket 


\section{Abstract}

\section{Background}

Semi-synthetic oleanane triterpenoid antioxidant inflammation modulators (tpAIMs) are small molecules that interact with KEAP1 cysteine residue 151 (C151) and activate NRF2. Exploration of the structureactivity relationship between the tpAIMs and KEAP1 is limited by the predominantly hydrocarbon nature of the oleanane triterpenoid pentacyclic ring structure. Therefore, we used novel, chemically tractable, synthetic antioxidant inflammation modulators (sAIMs) to probe the stereoselectivity of the ligandprotein interaction.

\section{Methods}

We measured several parameters of NRF2 activation to assess the potency of sAIM enantiomers with natural (tpAIM-like) $4(S), 5(S), 10(R)$ or unnatural 4(R),5(R),10(S) configurations. Additionally, we determined the crystal structure of the KEAP1 BTB domain in complex with two different sAIMs.

\section{Results}

We found that the potencies of sAIM enantiomers in the natural configuration were similar to those of the tpAIM, RTA 405. Strikingly, sAIM enantiomers in the unnatural configuration were 10- to 40-fold less potent than their natural counterparts. Crystallographic studies of sAIMs in complex with the KEAP1 BTB domain demonstrated that these ligands form a covalent bond with $\mathrm{C} 151$ and revealed the presence of additional hydrogen bonds, Van der Waals interactions, and pi-stacking interactions.

\section{Conclusions}

Although KEAP1 C151 is required for NRF2 activation by tpAIMs and sAIMs, interactions with other KEAP1 residues are critical for the stereospecific recognition and potency of these ligands.

\section{General Significance}

This work demonstrates that reversible cyanoenone Michael acceptors, such as the tpAIMs and sAIMs, can be specifically tuned to regulate redox sensitive cysteine residues on key signaling molecules, an approach with significant promise for innovative drug development. 
Keywords: Kelch-like ECH-associated protein 1 (KEAP1), Nuclear factor 2 (erythroid-derived 2-like factor) (NFE2L2 or NRF2), reactive cysteine residue, triterpenoid antioxidant inflammation modulator (tpAIM), synthetic antioxidant inflammation modulator AIM (sAIM), X-ray crystallography 


\section{Introduction}

Nuclear factor, erythroid 2-like 2 (NFE2L2, also known as NRF2), is a transcription factor that serves as a key regulator of the cellular response to oxidative and inflammatory stress. Under normal physiological conditions, Kelch-like ECH-associated protein 1 (KEAP1) maintains low levels of NRF2 by promoting its CUL3-RBX1-mediated ubiquitination and subsequent proteasomal degradation. Under stressful conditions, reactive oxygen and nitrogen species modify sensor cysteine residues within KEAP1 and block the degradation of NRF2. Newly synthesized NRF2 then translocates to the nucleus, binds to antioxidant response elements, and increases transcription of numerous genes that encode cellular defense and repair enzymes [1]. This coordinated and rapid upregulation of multiple genes ensures a robust response to many types of cellular stress.

The KEAP1/NRF2 pathway has emerged as a high-profile drug target due to accumulating evidence that supports NRF2's protective role in inflammation-associated disease [2;3]. Semi-synthetic oleanane triterpenoids, such as CDDO, CDDO-Me (also known as RTA 402 or bardoxolone methyl), and RTA 408 (also known as omaveloxolone) are among the most potent small molecule NRF2 activators identified to date [4;5]. These triterpenoid antioxidant inflammation modulators (tpAIMs) exhibit broad anti-inflammatory and cytoprotective activity in animal models of acute injury and chronic disease [6]. In the clinic, treatment with bardoxolone methyl increased NRF2 activity in peripheral blood mononuclear cells isolated from patients with advanced cancer [7], and increased estimated glomerular filtration rate in patients with chronic kidney disease (CKD) and type 2 diabetes [8;9]. Based on these promising results, the phase 3 Bardoxolone Methyl Evaluation in Patients with Chronic Kidney Disease and Type 2 Diabetes Mellitus: the Occurrence of Renal Events (BEACON) trial was initiated. However, in 2012, an imbalance in the rate of cardiac events $(8.8 \%$ in the bardoxolone methyl group vs. $5.0 \%$ in the placebo group) resulted in the premature termination of the trial [2]. Post hoc analyses revealed that the risk for fluid overload and heart failure was higher in bardoxolone methyl-treated patients that had specific, identifiable, risk factors, including prior hospitalization for heart failure and elevated serum levels of 
brain natriuretic peptide (BNP, a surrogate marker of fluid retention) [3]. In patients without these risk factors, the rate of heart failure was similar between the two treatment groups. Based on this manageable safety profile, the regulatory bodies in the US and Japan have allowed clinical testing of bardoxolone methyl to continue. The safety and efficacy of bardoxolone methyl is currently being evaluated in patients with pulmonary hypertension [10;11] and in patients with CKD and type 2 diabetes [12]. In addition, clinical trials of omaveloxolone are currently underway in several indications, including Friedreich's Ataxia [13], mitochondrial myopathies [14], corneal endothelial cell loss following cataract surgery [15], and melanoma [16]. The increased risk for acute fluid overload adverse events observed in BEACON with late-stage CKD patients has not been observed in subsequent studies with bardoxolone methyl or omaveloxolone.

The potency of the tpAIMs is dependent on the presence of a cyanoenone Michael acceptor in the A ring and a second enone in the $\mathrm{C}$ ring of the triterpenoid scaffold. Initial studies demonstrated that the A ring Michael acceptor forms reversible adducts with thiols $[4 ; 17 ; 18]$ and interacts with cysteine sulfhydryls in KEAP1 [4]. Of the twenty-seven cysteine residues in the human KEAP1 protein, threeC151, C273, and C288, which are conserved across species-are particularly important for the physiological function of KEAP1 [19]. Recent studies have revealed that chemical NRF2 inducers fall into four distinct groups based on their preference for specific cysteine residues [19-21]. Class I inducers [e.g., sulforaphane, diethyl maleate (DEM), and dimethyl fumarate (DMF), tert-butylhydroquinone (tBHQ)] prefer C151, which is located in the Broad-complex, Tramtrack, Bric-a-brac (BTB) domain of KEAP1. In contrast, class II inducers (e.g., 15d-PGJ 2 ) prefer C288, and Class III inducers (e.g., 4-HNE and $\mathrm{NaAsO}_{2}$ ) can utilize $\mathrm{C} 151, \mathrm{C} 273$, or $\mathrm{C} 288$. The activity of the fourth class of NRF2 inducers (e.g., $\mathrm{H}_{2} \mathrm{O}_{2}$ ) is not dependent on $\mathrm{C} 151, \mathrm{C} 273$, or $\mathrm{C} 288$; however, the sensor cysteine residues for these compounds have not been identified [20]. The tpAIMs have been identified as Class I NRF2 inducers; that is, their NRF2-inducing activity is mediated predominantly through KEAP1 C151 [20;22;23]. The covalent interaction between tpAIMs and C151 has recently been validated by a crystal structure of the KEAP1 BTB domain in complex with CDDO [24]. 
The tpAIMs are at least an order of magnitude more potent than other Class I NRF2 inducers [21], suggesting that molecular interactions between tpAIMs and KEAP1, beyond C151 ligation, influence the ability of the tpAIMs to inhibit KEAP1-mediated NRF2 degradation. With this in mind, we hypothesized that the mechanism of action of the tpAIMs is not mediated through indiscriminant oxidation of KEAP1 cysteine residues, but rather is dependent on transient ligation of a single cysteine residue on KEAP1 via highly specific ligand-protein interactions. However, it was not possible to test this hypothesis by modifying the tpAIMs because exploration of structure-activity relationship (SAR) is limited by the predominantly hydrocarbon nature of the oleanane triterpenoid pentacyclic ring structure. Of particular note, it is not possible to assess the role of stereoselective antipodal recognition —a hallmark of specific ligand-protein interaction — using the tpAIMs due to the solitary global stereochemical scaffold of the natural product. Therefore, to further our understanding of tpAIM SAR and to test whether stereospecific recognition of tpAIMs by KEAP1 contributes to their high potency, we synthesized novel, purely synthetic AIMs (sAIMs) and compared their antioxidant and anti-inflammatory activities to those of RTA 405, a well-characterized tpAIM.

We assessed both the natural (tpAIM-like) $4(S), 5(S), 10(R)$ and the unnatural $4(R), 5(R), 10(S)$ sAIM enantiomers to determine whether their anti-inflammatory and antioxidant activities were biased toward a specific configuration. Next, we determined whether the activities of the sAIMs, like the tpAIMs, are dependent on NRF2 and KEAP1 C151. Finally, we solved the crystal structures of the KEAP1 BTB domain in complex with two different natural sAIM enantiomers and characterized the molecular interactions between the ligands and the residues comprising the KEAP1 C151 binding site. The results presented herein clearly show that the potencies of the natural sAIM enantiomers and the tpAIMs are very similar, and that their activity is dependent on the presence of Nrf2. In addition, the KEAP1-sAIM crystal structures provide a rationale for the stereospecific recognition of tpAIMs by KEAP1. These findings hold considerable promise for the development of additional highly-specific, synthetic, KEAP1 C151-targeted Nrf2 activators for the treatment of a broad array of acute and chronic inflammatory and autoimmune diseases where oxidative and nitrosative stress plays a role. 


\section{Materials and methods}

\subsection{Materials}

RTA 405 (2-cyano-3,12-dioxooleana-1,9(11)-dien-28-oic acid ethyl amide), RTA 402 (methyl 2cyano-3,12-dioxoolean-1,9-dien-28-oate) and all sAIMs were synthesized by Reata Pharmaceuticals, Inc. (Irving, TX). The tpAIM C17-biotin conjugate probe was synthesized as previously described [25]. Stock solutions $(10 \mathrm{mM})$ of tpAIMs, sAIMs, and the biotinylated tpAIM probe were made in DMSO and stored at $-80^{\circ} \mathrm{C}$. Mouse recombinant IFN $\gamma$ was from RD Systems. Unless otherwise noted, all other chemicals were purchased from Sigma-Aldrich. Wild-type, C151S and $\mathrm{Keapl}^{-/-}$murine embryonic fibroblasts (MEFs) were obtained from the laboratory of Masayuki Yamamoto under a licensing agreement with Tohoku University, Japan [19;26]. The AREc32 reporter cell line was obtained from CXR Bioscience Limited (Dundee, UK). All other cell lines were from the American Type Culture Collection (ATCC). ATCC uses short tandem repeat analysis to screen all human cell lines for authenticity and purity before distribution. All cell lines were passaged for less than six months after resuscitation.

\subsection{Cell culture}

MEFs, HEK-293, and AREc32 cells were cultured in DMEM. RAW 264.7 cells were cultured in RPMI 1640 media. AML-12 cells were cultured in DMEM:F12 media. Unless otherwise noted, all media was from Life Technologies and was supplemented with 10\% FBS and 1\% penicillin/streptomycin. Medium for AREc32 cells also contained $0.8 \mathrm{mg} / \mathrm{ml}$ Geneticin (Life Technologies). Cell lines were cultured in the presence of $5 \% \mathrm{CO}_{2}$ at $37^{\circ} \mathrm{C}$. DMSO at $\leq 0.1 \%$ was used as a vehicle control in all experiments.

\subsection{Subcloning and site-directed mutagenesis}

Full-length human Keapl cDNA was obtained from Invitrogen (I.M.A.G.E. Consortium, Clone ID 3955118) and amplified by PCR using the following primers: 5'-tggggatccatgcagccagatcccag-3' and 
5'-aaaagcttatcaacaggtacagttctgctg-3'. The product was digested with BamHI and HindIII and cloned into a pET28a expression vector (Novagen, now EMD Millipore). A KpnI restriction enzyme site was introduced upstream of the NcoI site by site-directed mutagenesis using the following primers: 5'ctttaagaaggagaggtaccatgggcagcagcc-3' and 5'-ggctgctgcccatggtacctctccttcttaaag-3'. The KpnI/XhoI fragment was subcloned into the pCDNA3.1 mammalian expression vector (Life Technologies). The resulting construct (pCDNA3.1-6xHis/T7-KEAP1) encoded the full-length human KEAP1 protein (residues 1-624) with 6xHis- and T7-tags attached to the N-terminal end. Cysteine residues 151, 273, and 288 were individually mutated to serine residues using site-directed mutagenesis. To generate constructs for structural studies, cDNA encoding the BTB domain of human KEAP1 (residues 49-182) was amplified by PCR using the following primers: 5'-caggctagcaaccgcaccttcagctac-3' and 5'ccgaaagcttagctggggtccagctgct-3'. The product was digested with NheI and HindIII and subcloned into a pET28a vector. All DNA constructs were verified by sequencing.

\subsection{KEAP1 interaction with biotinylated tpAIM probe}

HEK-293 cells were plated in 6-well dishes at a density of $6 \times 10^{5}$ cells per well. The following day, cells were transfected with $1 \mu \mathrm{g}$ of wild-type, C151S, C273S, or C288S mutant pCDNA3.16xHis/T7-KEAP1 DNA per well using FuGENE 6 transfection reagent (Roche Applied Science) according to the manufacturer's protocol. Twenty-four hours after transfection, the culture media was removed and substituted with DMEM containing 1\% FBS. Two hours later, cells were treated with DMSO or $7.5 \mu \mathrm{M}$ biotinylated tpAIM probe for $2 \mathrm{~h}$. Following treatment, cells were washed with PBS and harvested in $200 \mu \mathrm{L}$ of ice-cold buffer $(50 \mathrm{mM}$ Tris $\mathrm{pH} 8.4,50 \mathrm{mM} \mathrm{NaCl}, 250 \mathrm{mM}$ sucrose, $2 \mathrm{mM}$ EDTA, 1 mM EGTA, 2 mM TCEP) supplemented with protease inhibitor cocktail (Roche Applied Science), and disrupted by sonication. Cell lysates were clarified by centrifugation at $18,000 \times \mathrm{g}$ for 10 min at $4{ }^{\circ} \mathrm{C}$. Equal volumes of total cell lysate were diluted 10-fold with binding buffer (PBS containing 0.4\% Tween-20, 2 mM TCEP, 2 mM EDTA, 1 mM EGTA and supplemented with protease inhibitors), and incubated overnight at $4^{\circ} \mathrm{C}$ with $100 \mu \mathrm{L}$ NeutrAvidin resin (ThermoScientific). The resin was 
collected by centrifugation for $5 \mathrm{~min}$ at 3,000 $\mathrm{x} g$ at $4^{\circ} \mathrm{C}$, and washed 5 times with $1 \mathrm{~mL}$ binding buffer. Bound material was eluted in SDS-PAGE sample buffer, and analyzed by electrophoresis on an 8\% SDS polyacrylamide gel followed by immunoblotting with T7 antibody (Novagen, now EMD Millipore).

\section{5. qPCR}

Wild-type, KEAP1-null and KEAP1-C151S MEFs were plated in 6-well dishes at densities of $3 \mathrm{x}$ $10^{5}, 1.5 \times 10^{5}$, and $3 \times 10^{5}$ cells per well, respectively, and treated for $18 \mathrm{~h}$ with DMSO, TX63541, or RTA 405. RAW 264.7 cells were plated in 6-well culture dishes at a density of $5 \times 10^{5}$ cells per well in RPMI 1640 supplemented with $0.5 \%$ FBS. The following day, RAW 264.7 cells were pretreated with DMSO, RTA 405, or TX63541 for $2 \mathrm{~h}$ and then treated with $20 \mathrm{ng} / \mathrm{ml} \mathrm{IFN} \gamma$ for $24 \mathrm{~h}$.

Following treatment, RNA isolation and reverse transcription were performed as described in [5]. Quantitative PCR was performed using Power SYBR Green Master Mix with ROX (Life Technologies) in a Viia7 Real Time PCR Detection System (Life Technologies). All PCR primers (Table 1) were

Table 1. Mouse PCR primers.

\begin{tabular}{lll}
\hline Gene Symbol & \multicolumn{1}{c}{ Forward primer } & \multicolumn{1}{c}{ Reverse primer } \\
Gclm & 5'-GCCACCAGATTTGACTGCCTTTG-3' & 5'-TGCTCTTCACGATGACCGAGTACC-3' \\
iNos (Nos2) & 5'-ATTGCTCCCTTCCGAAGTTT-3' & 5'-TGCAGGATGTCCTGAACGTA-3' \\
Mcp1 (Cc12) & 5'-GCAGAGAGCCAGACGGGAGGA-3' & 5'-TCAGGATGGTTTGCAATGAA-3' \\
Nqo1 & 5'-TCGGGCTAGTCCCAGTTAGA-3' & 5'-AAAGAGCTGGAGAGCCAACC-3' \\
Nrf2 (Nfe212) & 5'-TCGGGCTAGTCCCAGTTAGA-3' & 5'-AAAGAGCTGGAGAGCCAACC-3' \\
Rpl19 & 5'-TCAGGCTACAGAAGAGGCTTGC-3' & 5'-ACAGTCACAGGCTTGCGGATG-3' \\
Txnrd1 & 5'-AGGAGAAAGGACCAGGGAAA-3', & 5'-TCCTTCTTGTGGGGGTAGAA-3' \\
\hline
\end{tabular}

previously validated for specificity and amplification efficiency. Viia7 and GraphPad Prism software were used to analyze the data. The relative abundance of each target gene was determined by normalizing to vehicle-treated samples (for MEFs) or vehicle/IFN $\gamma$-treated samples (for RAW 264.7 cells) using the Livak method of analysis $\left(2^{-\Delta \Delta \mathrm{CT}}\right)$ [27]. The error (range) of the technical and experimental replicates was 
calculated using the following equations: upper limit $=2^{-[\Delta \Delta \mathrm{CT}-\mathrm{SD}(\Delta \Delta \mathrm{CT})]}$, lower limit $=2^{-[\Delta \Delta \mathrm{CT}+\mathrm{SD}(\Delta \Delta \mathrm{CT})]}$. Ribosomal protein L19 (Rpl19) served as an endogenous control gene.

\subsection{Western blots}

Wild-type, KEAP1-null, and KEAP1-C151S MEFs were plated in 10-cm dishes. The following day, cells were treated with DMSO or 100 nM RTA 405 for 24 h. Cells were collected, washed with PBS, and resuspended in $300 \mu \mathrm{L}$ hypotonic buffer A (10 mM HEPES, $10 \mathrm{mM} \mathrm{KCl}, 1.5 \mathrm{mM} \mathrm{MgCl} 2,1 \mathrm{mM}$ DTT, pH 7.4, supplemented with protease inhibitors (Roche Applied Science)). After 15 min on ice, $18.75 \mu 1$ of $10 \%$ NP-40 was added, samples were briefly vortexed, and nuclear fractions were separated by centrifugation at $3,000 \times \mathrm{g}$ for $5 \mathrm{~min}$ at $4^{\circ} \mathrm{C}$. Protein concentration in the supernatant (cytosolic fraction) was determined using DC protein assay (Bio-Rad). Cytosolic proteins (30 $\mu \mathrm{g})$ were separated on an $8 \%$ polyacrylamide tricine gel, transferred to nitrocellulose membranes, and incubated with the indicated antibodies overnight.

RAW 264.7 cells were plated in 24-well culture dishes at a density of $1.5 \times 10^{5}$ cells per well in RPMI 1640 supplemented with 0.5\% FBS. The following day, cells were pretreated with DMSO or the indicated concentrations of test compounds for $2 \mathrm{~h}$, then treated with $20 \mathrm{ng} / \mathrm{mL}$ IFN $\gamma$ for $24 \mathrm{~h}$. Cells were washed with sterile PBS and lysed in tricine sample buffer with $2 \% \beta$-mercaptoethanol (BME). For IкB $\alpha$ and phospho-I $\mathrm{B} \mathrm{B} \alpha$ western blots, RAW 264.7 cells were plated in 6-well culture dishes at a density of $1 \times 10^{6}$ cells per well in RPMI 1640 supplemented with $0.5 \%$ FBS. The next day, cells were pretreated with RTA405, TX63541, or DMSO for $24 \mathrm{~h}$, then treated with $20 \mathrm{ng} / \mathrm{mL}$ IFN $\gamma$ for $3 \mathrm{~h}$. Cells were washed with sterile PBS and lysed in tricine sample buffer with $1 \% \beta$-mercaptoethanol (BME). For nuclear NRF2, cell lysates were fractionated using a Nuclear Extract Kit (Active Motif). Whole cell or fractionated lysates were heated at $100^{\circ} \mathrm{C}$ for $30 \mathrm{~min}$ and separated on $10 \%$ polyacrylamide tricine gels, then transferred to nitrocellulose membranes, and incubated with the indicated antibodies overnight.

Antibodies and dilutions used were: NQO1 (Abcam, ab23461, 1:1000), iNOS (Santa Cruz, sc650, 1:200), GCLM (Abcam, 5529-1, 1:2000), NRF2 (Santa Cruz, sc13032, 1:500 ), Lamin B (Santa 
Cruz, sc6217, 1:500), GAPDH (Sigma-Aldrich, SAB2500451, 1:500), IкB $\alpha$ (Santa Cruz, sc371, 1:500), pI $\mathrm{B} \alpha$ (Cell Signaling Technologies, 9246, 1:400), and Actin (Millipore, MAB1501, 1:40,000 for RAW 264.7 cells and 1:100,000 for MEFs). Secondary antibodies were from Jackson ImmunoResearch.

\subsection{Nitric oxide assay (Griess reaction)}

RAW 264.7 mouse macrophages were plated in 96-well culture dishes in triplicate wells at a density of $3.0 \times 10^{5}$ cells per well in RPMI 1640 supplemented with $0.5 \%$ FBS. The following day, cells were pre-treated with DMSO or test compounds for $2 \mathrm{~h}$. Cells were then treated with $20 \mathrm{ng} / \mathrm{ml} \mathrm{IFN \gamma}$ for 24 h. Nitrite $\left(\mathrm{NO}_{2}{ }^{-}\right)$levels in media were measured as described in [5]. $\mathrm{IC}_{50}$ values were calculated using GraphPad Prism.

\subsection{AREc32 luciferase reporter assay}

AREc32 cells were plated in solid black 384 -well plates in $50 \mu \mathrm{L}$ media at a density of $5 \times 10^{3}$ cells per well. The following day, cells were treated with DMSO or test compound for $19 \mathrm{~h}$. After a 15min equilibration at room temperature, $50 \mu \mathrm{L}$ ONE-Glo Luciferase reagent (Promega) was added to each well for 5 min at room temperature. Luminescence was measured using a BMG PheraStar microplate reader. Firefly luciferase activity in each compound-treated sample was normalized to vehicle control. Dose-response curves were generated using the log(agonist) vs. response -- variable slope (four parameters) equation in GraphPad Prism (San Diego, CA). The concentration of compound that increased reporter activity by 2 -fold $\left(\mathrm{EC}_{2 \mathrm{x}}\right.$ value) was extrapolated from the curve. Cell viability was monitored in parallel using Cell Proliferation Reagent WST-1 (Roche Applied Science) (data not shown).

\subsection{ELISA}

RAW 264.7 cells were plated in 6-well culture dishes at a density of $5 \times 10^{5}$ cells per well in RPMI 1640 supplemented with 0.5\% FBS. The following day, cells were pretreated with DMSO, RTA 405, or TX63541 for $2 \mathrm{~h}$ and then treated with $20 \mathrm{ng} / \mathrm{mL}$ IFN $\gamma$ for $24 \mathrm{~h}$. Culture media was collected from 
the plates and centrifuged at $4^{\circ} \mathrm{C}$ for $20 \mathrm{~min}$. Supernatant was diluted 1:50 and the MCP1 ELISA assay (RD Systems, MJE00) was performed according to the manufacturer's protocol. Absorbance was measured at 450 and $540 \mathrm{~nm}$ using a BMG PheraStar plate reader. MCP1 concentrations were determined by extrapolation from a standard curve.

\subsection{SiRNA}

RAW 264.7 cells were reverse transfected in OptiMEM with Lipofectamine RNAiMax (Life Technologies) and $10 \mathrm{nM}$ siNRF2 or $10 \mathrm{nM}$ siNTPool (Dharmacon, L-040766-00-0005 and D-00181-1005, respectively). Mock-transfected samples did not receive siRNA. Cells were plated in 24-well plates at a density of $2 \times 10^{5}$ cells per well or 6-well plates at a density of $1 \times 10^{6}$ cells per well in RPMI 1640 media supplemented with $0.5 \%$ FBS. Media was changed after $48 \mathrm{~h}$ and cells were pretreated with DMSO or RTA 405, or TX63541 for $2 \mathrm{~h}$. Cells were then treated with $20 \mathrm{ng} / \mathrm{mL}$ IFN $\gamma$ for $2 \mathrm{~h}$ (nuclear NRF2) or $24 \mathrm{~h}$ (all other experiments). Supernatant was collected from the 6-well plates for the nitric oxide assay and MCP1 ELISA, each performed as described above. The remaining cells in the 6-well plates were harvested in RNeasy RLT Buffer for reverse transcription and qPCR as described above for RAW 264.7 cells. Cells from the 24-well plates were washed with sterile PBS and harvested for western blots as described above for RAW 264.7 cells. The relative abundance of each target gene was determined by normalizing to vehicle-treated mock-transfected samples (for Nrf2, Nqol, Gclm, and Txnrd1) or vehicle/IFN $\gamma$-treated mock-transfected samples (iNos and Mcpl) using the Livak method of analysis ( $2^{-}$ $\Delta \Delta \mathrm{CT})[27]$.

\subsection{Statistical analyses of biological data}

Statistical analyses of biological data were performed prior to normalization relative to the control sample (i.e. raw data). For AREc32 luciferase reporter assays, luminescence values were used; for qPCR experiments, $\Delta \mathrm{C}_{\mathrm{T}}$ values were used; for ELISA experiments, protein concentrations were used. For all dose-response experiments, the statistical significance of the effect of compound treatment was 
determined using one-way ANOVA and Dunnett's multiple comparison test. For siRNA experiments, the statistical significance of the difference between siRNA treatment groups (i.e., Mock vs. Nrf2 and Nontargeting vs. Nrf2) was determined using the paired t-test at each concentration of compound tested.

\subsection{Protein co-expression and co-purification with a ligand}

KEAP1 BTB 49-182 was expressed in BL21-CodonPlus (DE3)-RIL E. coli (Agilent Technologies). A 30-mL overnight culture in LB/Kanamycin (Kan) was centrifuged at 4,000 rpm for 5 min at $25^{\circ} \mathrm{C}$ and the pellet was resuspended in $15 \mathrm{~mL}$ minimal media $\left(\mathrm{MM} ; 18.7 \mathrm{mM} \mathrm{NH}_{4} \mathrm{Cl}, 7.3 \mathrm{mM}\right.$

$\mathrm{KH}_{2} \mathrm{PO}_{4}, 11.2 \mathrm{mM} \mathrm{K}_{2} \mathrm{HPO}_{4}, 2.1 \mathrm{mM} \mathrm{Na}_{2} \mathrm{SO}_{4}, 0.246 \mathrm{mM} \mathrm{MgCl}_{2}$, and $0.034 \mathrm{mM} \mathrm{CaCl}_{2}, 0.3 \%$ glucose, and $30 \mu \mathrm{g} / \mathrm{mL}$ Kan) and added to $1 \mathrm{~L} \mathrm{MM} / \mathrm{Kan}$. Culture was incubated at $37^{\circ} \mathrm{C} / 250 \mathrm{rpm}$, these parameters were reduced to $16^{\circ} \mathrm{C} / 180 \mathrm{rpm}$ at $\mathrm{OD}_{600}=0.4-0.6$. After $1 \mathrm{~h}$ equilibration, $0.02 \mathrm{mM}$ of sAIM ligand (0.2\% final DMSO) was added to the culture for $30 \mathrm{~min}$ followed by the induction of protein expression with $0.4 \mathrm{mM}$ IPTG for an additional 16-18 h.

Cells were harvested by centrifugation at $4,000 \mathrm{rpm}$ for $30 \mathrm{~min}$ at $4^{\circ} \mathrm{C}$ and the pellet was resuspended in 100 mL Lysis Buffer (Buffer-A: 20 mM HEPES, 300 mM NaCl, 40 mM imidazole, pH 7.5) supplemented with $100 \mathrm{mM}$ AEBSF and 2 tablets of EDTA-free Protease Inhibitor Cocktail (Roche). Prior to sonication, suspension was treated with $0.2 \mathrm{mg} / \mathrm{mL}$ lysozyme for $25 \mathrm{~min}$ at room temperature with gentle shaking, followed by freeze/thaw with liquid nitrogen and lukewarm tap water, respectively. Lysate was centrifuged at $30,000 \mathrm{x} g$ for $30 \mathrm{~min}$ at $4^{\circ} \mathrm{C}$ and supernatant was treated with $5 \mathrm{~mL} \mathrm{Ni}$ sepharose 6 fast flow resin (GE Healthcare), equilibrated with Buffer-A, for $1 \mathrm{~h}$ with periodic resuspension of resin. Gravity purification was performed using step elution with the Ni-resin washed with 10 column volumes of Buffer-A after flow-through was collected. 6xHis-tag BTB 49-182 was eluted with 5 column volumes of $100 \mathrm{mM}$ imidazole, $250 \mathrm{mM}$ imidazole, and $500 \mathrm{mM}$ imidazole added sequentially. Imidazole concentrations were achieved by mixing Buffer-A and Buffer-B (Buffer-A with $500 \mathrm{mM}$ imidazole). The $250 \mathrm{mM}$ imidazole eluate was dialyzed twice with $1 \mathrm{~L}$ Buffer-C (20 mM HEPES, $5 \mathrm{mM} \mathrm{NaCl}, 10 \mathrm{mM}$ imidazole, $2.5 \mathrm{mM}$ calcium chloride, $\mathrm{pH}$ 7.5). Each dialysis was performed 
for $1.5 \mathrm{~h}$ at $4^{\circ} \mathrm{C}$ without stirring. Sample was filtered and treated with $50 \mathrm{U}$ Thrombin overnight (14-16 h) at $4^{\circ} \mathrm{C}$ to remove $6 x$ His-tag. Cleavage with thrombin results in a short vector-derived amino acid sequence (GSHMAS) that precedes the BTB domain.

A second Ni-sepharose gravity purification was performed to capture the 6xHis-tag, where sample was treated with $2 \mathrm{~mL}$ Ni resin, equilibrated with Buffer-C, for 30 min with periodic suspension, and flow-through containing BTB 49-182 was collected. BTB 49-182 was further purified by Mono Q 10/100 GL affinity column (GE Healthcare) equilibrated with Buffer-D (20 mM HEPES, 5 mM NaCl, pH 7.5) and eluted with a 0\%-50\% linear elution gradient using Buffer-E (20 mM HEPES, $1 \mathrm{M} \mathrm{NaCl}, \mathrm{pH}$ 7.5), followed by size exclusion chromatography (SEC) using a Superdex 200 16/60 HiLoad column (GE Healthcare) equilibrated with Buffer-F (20 mM HEPES, $150 \mathrm{mM} \mathrm{NaCl}, \mathrm{pH}$ 7.5). The BTB 49-182/sAIM complex was concentrated, filtered through a $0.22 \mu \mathrm{m}$ filter and final protein concentration was determined using a protein assay kit (Bio-Rad). Protein purity was monitored by SDS-PAGE. All buffers contained $2 \mathrm{mM}$ TCEP and $0.02 \mathrm{mM}$ sAIM ligand (0.2\% DMSO), with ligand added to buffers at room temperature prior to chilling to $4^{\circ} \mathrm{C}$. Mono Q and SEC buffers were filtered and degassed prior to addition of ligand.

\subsection{Buffer and compound exchange}

Co-expression and co-purification of BTB 49-182 with TX64014 resulted in crystals of low resolution X-ray diffraction. To improve the resolution of X-ray diffraction, previously co-expressed and co-purified BTB 49-182/TX64014 complex ( 1 mM) was thawed, diluted 25-times with Buffer-F at pH 8.0 with $0.02 \mathrm{mM}$ TX64014 (0.2\% DMSO), and treated with an additional $0.2 \mathrm{mM}$ of TX64014 (2\% DMSO final) for $2 \mathrm{~h}$ at room temperature. The sample was concentrated and re-purified by SEC using Superdex 200 10/300 GL column equilibrated with pH modified Buffer-F. 


\subsection{Crystallization and structure determination}

Multiple high-throughput crystallization screens were used for both complexes where $0.2 \mu \mathrm{L}$ of protein is mixed with $0.2 \mu \mathrm{L}$ of crystallization condition (Art Robbins Instruments) and equilibrated against a $50 \mu \mathrm{L}$ reservoir at $20^{\circ} \mathrm{C}$ via vapor diffusion in a sitting drop. Hexagonal rod crystals appeared within two days for the BTB 49-182-TX64014 complex at $11.1 \mathrm{mg} / \mathrm{mL}$ from Index crystallization screen well H11 (0.1 M Potassium thiocyanate and 30\% w/v PEG MME 2000; Hampton Research) and within one day for the BTB 49-182-TX64063 complex at $6.33 \mathrm{mg} / \mathrm{mL}$ from Wizard 1 and 2 crystallization screen well D5 (0.1 M CHES, pH 9.5 and 30\% w/v PEG 3000; Emerald Biosystems). Crystals from the screens were cryoprotected in crystallization condition buffer containing $30 \% \mathrm{w} / \mathrm{v}$ glucose and flash frozen with liquid nitrogen.

Data collection and processing was performed at the IMCA-CAT beamline 17-ID at APS-ANL with a resolution cutoff at $2.61 \AA$ for BTB 49-182-TX64014 complex and 2.37 A for BTB 49-182TX64063 complex. Phases were determined by molecular replacement using Phaser [28]. Structure of human KEAP1 BTB domain (residues 39-179) in which cysteine 151 was mutated to serine was used as the search model for the BTB 49-182-TX64063 complex dataset, and BTB 49-182 was used as the search model for the BTB 49-182-TX64014 complex dataset. The mutant KEAP1 BTB structure was solved by molecular replacement using coordinates from the structure of the transcriptional regulator LRF/ZBTB7 (PDB ID: 2NN2) as the search model (www.globalphasing.com). Refinement was performed with Refmac [29] using isotropic B factor restrained refinement with the matrix weight being relaxed at the final stages of refinement. Visualization and manual model building was performed with Coot [30], with waters added manually. Crystallographic information files (CIF) for C151 adduct to TX64014 and TX64063 were generated with JLigand [31]. Phaser and Refmac are part of the CCP4 suite [32]. All images of structures, electron density maps and electrostatic surface potentials were rendered using PyMOL (Schrödinger, LLC). Atomic coordinates and structure factors were deposited with the Protein Data Bank (PDB). PDB ID: 5DAD for the KEAP1 BTB-TX64014 complex and PDB ID: 5DAF for the KEAP1 BTB-TX64063 complex. 


\section{Results}

\subsection{KEAP1 cysteine residue 151 is required for RTA 405 activity}

Previous studies have shown that KEAP1 C151 is required for NRF2 activation by the tpAIMs, CDDO-Im and dh404 [19;20;22;23]. To evaluate whether C151 is also necessary for tpAIMs to bind to KEAP1, we transfected HEK-293 cells with expression constructs containing either the wild-type or one of three mutant (C151S, C273S, or C288S) KEAP1 sequences and performed pull-down experiments with a biotinylated tpAIM probe (Fig. 1A). Equivalent amounts of the wild-type, C273S, and C288S KEAP1 proteins were pulled down by the tpAIM probe (Fig. 1B). In contrast, the amount of C151S mutant KEAP1 protein pulled down by tpAIM probe was greatly reduced (Fig. 1B). This indicates that $\mathrm{C} 151$, but not $\mathrm{C} 273$ or $\mathrm{C} 288$, mediates binding of the tpAIM probe, which is consistent with the requirement of C151 for NRF2 activation by CDDO-Im and dh404. To determine whether RTA 405mediated NRF2 activation also requires C151, we measured the expression levels of a well-characterized NRF2 target gene, $N A D(P) H$ dehydrogenase, quinone 1 (Nqol), in wild-type, KEAP1-null, and KEAP1C151S MEFs. Consistent with published data [19], basal Nqo1 mRNA levels were elevated in KEAP1null MEFs relative to both wild-type and KEAP1-C151S MEFs (Fig. 1C). Treatment with RTA 405 dosedependently increased Nqol expression in wild-type MEFs, but not in KEAP1-null or KEAP1-C151S MEFs (Fig. 1D). We observed a similar pattern for NQO1 protein levels. Relative to wild-type and KEAP1-C151S MEFs, KEAP1-null MEFs had elevated basal NQO1 levels (Fig. 1E). Treatment with 100 nM RTA 405 increased NQO1 protein levels in wild-type MEFs, but not in KEAP1-null or KEAP1 C151S MEFs (Fig. 1E). Taken together, our results demonstrate that RTA 405-mediated activation of NRF2 requires KEAP1 C151.

\subsection{Design, synthesis, and biological activity of the synthetic AIMs}

We developed the sAIMs to probe the magnitude of the contribution of ligand structure topology to KEAP1 binding irrespective of cysteine ligation, and moreover to establish an avenue for manipulation 
A

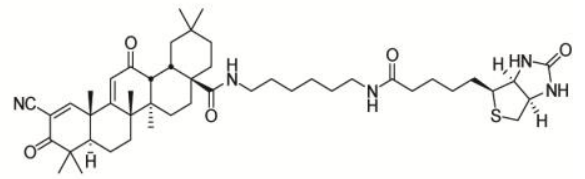

B

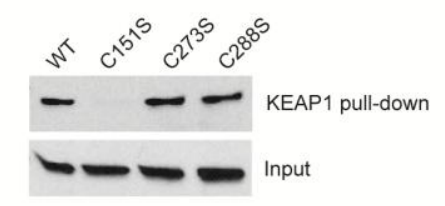

C

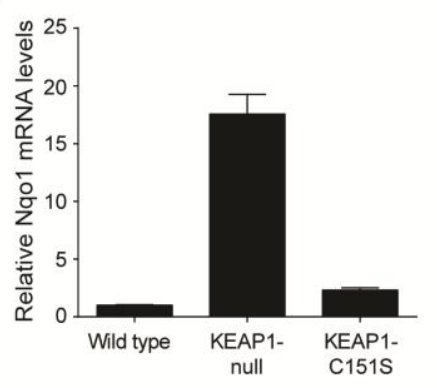

D

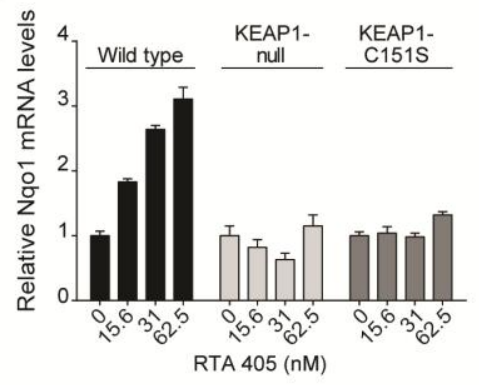

E

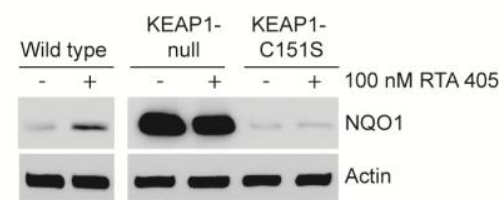

FIGURE 1. KEAP1 cysteine residue 151 is required for tpAIM activity. A. Structure of the biotinylated tpAIM probe. B. Pull-down assay with biotinylated tpAIM probe. HEK-293 cells transfected with wildtype or mutant T7-tagged KEAP1 expression constructs were incubated with the biotinylated probe for 2 h. NeutrAvidin precipitations were performed as described in Materials and methods. Equal volumes of cell lysates (lower panel) and resin eluates (upper panel) were analyzed by Western blotting with T7 antibody. C. Basal Nqol mRNA levels were measured by qPCR in wild-type, KEAP1-null and KEAP1C151S MEFs. Nqo1 mRNA levels were normalized to wild-type (set to 1). Error bars represent the range of technical replicates. D-E. Wild-type, KEAP1-null and KEAP1-C151S MEFs were treated with DMSO or RTA 405 for 24 h. D. Nqol mRNA levels were evaluated by quantitative real-time PCR analysis and normalized to the DMSO-treated sample for each genotype (set to 1). Error bars represent the range of technical replicates. E. NQO1 protein levels were evaluated by western blot after resolving equal amounts of cytosolic protein by SDS-PAGE. Actin served as a loading control. All data are representative of two independent experiments. 
and improvement of the pharmaceutical properties of this important and unique class of NRF2 activators. The design concept retained certain key elements previously shown to be essential for high potency NRF2 activation [33], while introducing features which would readily facilitate expansion of the SAR beyond the tight restrictions imposed by the tpAIM hydrocarbon backbone (Fig. 2A). The elements of design held invariant were the bicyclic transfused decalin A,B ring structure including the potent and highly reversible 2-cyanoenone Michael acceptor and the critical apical C-10 methyl substituent which imparts strict facial selectivity for the addition of an activated cysteine to the beta carbon of the cyanoenone Michael acceptor. These features clearly arbitrate binding mode orientation and allow a rational assessment of the topological features influencing ligand/protein binding. Finally, appending either a pyrazole or pyrimidine heterocyclic C-ring variant completes the sAIM design and allows manipulation of physical properties and the introduction of much needed structural diversity. Importantly, the synthesis which proceeded through the Wieland-Miescher ketone afforded unequivocal access to both sAIM antipodes.

The anti-inflammatory activity of the pyrazole and pyrimidine sAIMs was evaluated by measuring suppression of interferon gamma-induced nitric oxide (NO) levels in the RAW 264.7 mouse macrophage cell line, an assay that has previously been used to assess the potency of the tpAIMs [34]. The racemic sAIMs, as exemplified by the pyrimidine TX63718 and the pyrazole TX63541 (Fig. 2B), were among the most potent compounds in this assay, with NO suppression potencies similar to RTA 405 and bardoxolone methyl (Fig. 3A and Table 2). In the AREc32 NRF2 reporter cell line [35], TX63718 and TX63541 increased NRF2 activity in a dose-dependent manner (Fig. 3B). The concentrations of sAIMs required to increase NRF2 activity by 2-fold $\left(\mathrm{EC}_{2 \mathrm{X}}\right)$ were similar to those of bardoxolone methyl and RTA 405 (Table 2). Moreover, the sAIM concentrations that are required for the anti-inflammatory (NO suppression) and antioxidant (NRF2 activation) activities are comparable and consistent with a mechanism whereby Nrf2 activation is required for NO suppression, as is observed for the tpAIMs [4]. Taken together, these results not only clearly validate the fidelity of our mimetic design but also provide 
key insights into the binding requirements proximal to the cyanoenone Michael acceptor, an important observation for future NRF2 activator optimization.

A Oleanane tpAIM
(natural configuration)

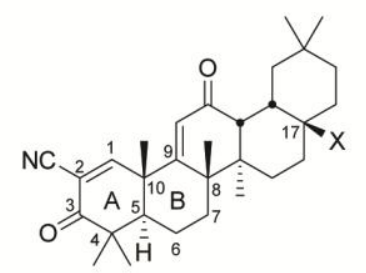

RTA 402: $\mathrm{X}=\mathrm{CO}_{2} \mathrm{CH}_{3}$

RTA 405: $\mathrm{X}=\mathrm{CONHC}_{2} \mathrm{H}_{5}$

B

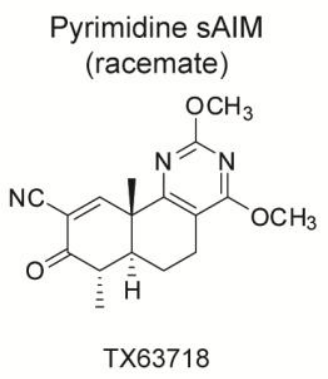

\section{Pyrazole sAIM} (racemate)<smiles>[R16]O[Z11]([H])([H])C</smiles>

Pyrimidine SAIM

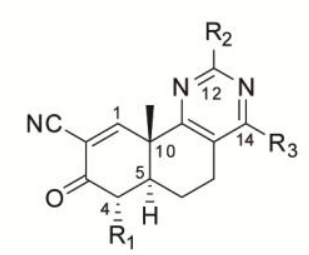

Pyrimidine sAIM (natural configuration)

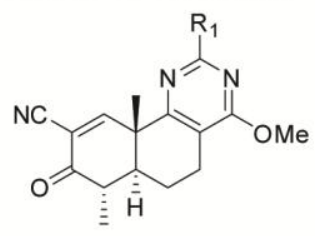

TX63793: $\mathrm{R}_{1}=\mathrm{OCH}_{3}$ TX64014: $\mathrm{R}_{1}=\mathrm{CH}_{3}$
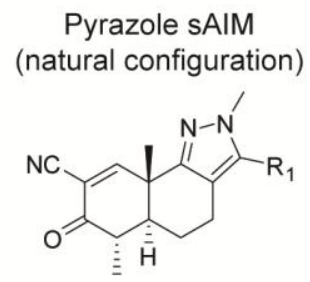

TX63791: $R_{1}=$ Phenyl TX64063: $R_{1}=3$-Pyridyl
Pyrazole sAIM<smiles>[R3]c1c2c(nn1[R])[C@@]1(C)C=C(C#N)C(=O)C([R1])[C@H]1CC2</smiles>

Pyrimidine SAIM (unnatural configuration)<smiles>[R]c1nc(OC)c2c(n1)[C@H]1C=C(C#N)C(=O)[C@H](C)[C@H]1CC2</smiles>

TX63794: $\mathrm{R}_{1}=\mathrm{OCH}_{3}$ TX285317: $\mathrm{R}_{1}=\mathrm{CH}_{3}$

Pyrazole sAIM (unnatural configuration)<smiles>[R]c1c2c(nn1C)[C@@]1(C)C=C(C#N)C(=O)C(C)[C@]1(C)CC2</smiles>

TX63792: $R_{1}=$ Phenyl TX70990: $R_{1}=3$-Pyridyl

FIGURE 2. Design and synthesis of the synthetic AIMs (sAIMs). A. Principle for the design of the sAIM pyrimidine and pyrazole mimetics based upon the structure of oleanane tpAIMs such as RTA 402 (bardoxolone methyl) and RTA 405. 1) The A-ring cyanoenone Michael acceptor, the C-10 apical methyl group and the transfused [6.6] decalin ring system were all held invariant. 2) Introduction of a chiral center at C-4 accompanied by either a pyrimidine or pyrazole fused C-ring completes the mimetic design. 3) Modification at $R_{1}, R_{2}$ and $R_{3}$ allow comprehensive exploration of the structure-activity-relationship for interaction of the sAIM mimetics with KEAP1. B. Chemical structures of sAIMs characterized in this study. 
Both TX63541 and TX63718 are racemic mixtures, nonetheless their relative configurations at C4, C-5, and C-10 were unambiguously established during synthesis due to the thermodynamically preferred 1,3 equatorial/axial relationship for the methyl groups at $\mathrm{C}-4$ and $\mathrm{C}-10$. To interrogate the activity differences of the sAIM enantiomers, we separated the racemic mixtures into the C-4(S), C-5(S), C-10 $(R)$ (natural) and C-4 $(R), \mathrm{C}-5(R), \mathrm{C}-10(S)$ (unnatural) configurations (Fig. 2B). Notably, the natural configuration is equivalent to the configuration of the natural product triterpenoid backbone of RTA 405 and bardoxolone methyl. A subsequent independent chiral synthesis of the natural enantiomer of TX63541, i.e., TX63791, unequivocally established the absolute stereochemistry as C-4(S), C-5(S) and C$10(R)$. When we evaluated the activity of these compounds in the NO suppression and NRF2 activation assays we found that the natural enantiomers (TX63791 and TX63793) were approximately twice as potent as their respective racemic mixtures, whereas the potencies of the unnatural enantiomers (TX63792 and TX63794) were greatly reduced (Table 2). This pattern of stereoselectivity is not unique to these two examples; it has been consistently observed with all high potency enantiomeric pairs of sAIMs that we have profiled to date. Two additional examples of the stereoselective activity are shown in Table 2. Unquestionably, these results clearly demonstrate that, although cysteine ligation plays an important role in NRF2 activation, the shape of the KEAP1 C151 binding site, as well as additional molecular interactions between the sAIMs and KEAP1, contribute significantly to the potency and selectivity of the natural enantiomer sAIMs. These data are not only highly supportive of potent and stereoselective ligation of KEAP1 by the sAIMs, but are also quite suggestive of a similar specific molecular recognition by the tpAIM derivatives. 

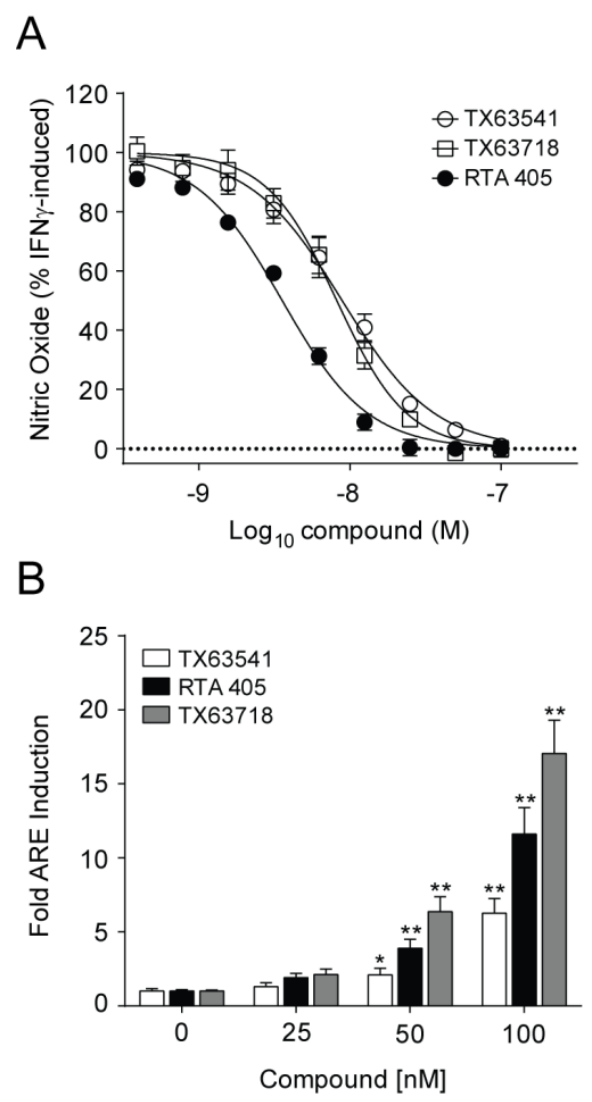

FIGURE 3. Antioxidant and anti-inflammatory effects of pyrimidine and pyrazole sAIMs. A. RAW 264.7 macrophages were pretreated with the indicated concentrations of RTA 405 (tpAIM), TX63541 (pyrazole sAIM), or TX63718 (pyrimidine sAIM) for $2 \mathrm{~h}$ and then treated with IFN $\gamma$ for an additional 24 h. Nitric oxide was measured to assess anti-inflammatory activity. Data are the average and SD of three independent experiments and are shown as percentage of the DMSO/IFN $\gamma$-treated sample. B. To assess NRF2 activity, AREc32 luciferase reporter cells were treated with the indicated concentrations of RTA 405, TX63541, or TX63718 for $19 \mathrm{~h}$. Data are the average and SD of three independent experiments, shown as fold-induction relative to DMSO-treated samples. ${ }^{*} P<.05$, $* * P<.01$ compared to the corresponding DMSO-treated sample for each compound. 
Table 2. Biological activity of sAIM enantiomers.

\begin{tabular}{|c|c|c|c|}
\hline \multirow[t]{2}{*}{ Compound ID } & \multirow[t]{2}{*}{ Enantiomer Configuration } & $\begin{array}{c}\text { Suppression of } \\
\text { IFN } \gamma \text {-induced } \\
\text { NO production }\end{array}$ & \multirow{2}{*}{ 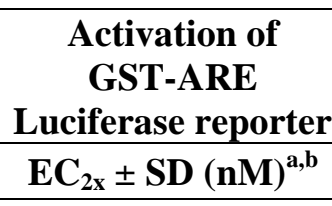 } \\
\hline & & $\mathrm{IC}_{50} \pm \mathrm{SD}(\mathrm{nM})^{\mathrm{a}}$ & \\
\hline \multicolumn{4}{|l|}{ tpAIMs } \\
\hline RTA 405 & $\mathrm{C} 4(S), \mathrm{C} 5(S), \mathrm{C} 10(R)$ & $4.0 \pm 1.2$ & $28.0 \pm 1.5$ \\
\hline Bardoxolone methyl & $\mathrm{C} 4(S), \mathrm{C} 5(S), \mathrm{C} 10(R)$ & $1.9 \pm 0.8 \mathrm{nM}^{\mathrm{c}}$ & $37.8 \pm 3.4$ \\
\hline \multicolumn{4}{|l|}{ Pyrazole sAIMs } \\
\hline TX63541 & Racemate & $13.0 \pm 1.4$ & $27.7 \pm 4.6$ \\
\hline TX63791 & $\mathrm{C} 4(S), \mathrm{C} 5(S), \mathrm{C} 10(R)$ (natural) & $7.7 \pm 0.9$ & $17.9 \pm 5.1$ \\
\hline TX63792 & $\mathrm{C} 4(R), \mathrm{C} 5(R), \mathrm{C} 10(S)$ (unnatural) & $186.6 \pm 5.4$ & $174.0 \pm 23.2$ \\
\hline TX64063 & $\mathrm{C} 4(S), \mathrm{C} 5(S), \mathrm{C} 10(R)$ (natural) & $16.2 \pm 1.9$ & $11.3 \pm 2.9$ \\
\hline TX70990 & $\mathrm{C} 4(R), \mathrm{C} 5(R), \mathrm{C} 10(S)$ (unnatural) & $685.9 \pm 34.9$ & $361.8 \pm 3.8$ \\
\hline \multicolumn{4}{|l|}{ Pyrimidine sAIMs } \\
\hline TX63718 & Racemate & $7.2 \pm 2.2$ & $24.2 \pm 2.4$ \\
\hline TX63793 & $\mathrm{C} 4(S), \mathrm{C} 5(S), \mathrm{C} 10(R)$ (natural) & $4.7 \pm 1.0$ & $9.2 \pm 2.2$ \\
\hline TX63794 & $\mathrm{C} 4(R), \mathrm{C} 5(R), \mathrm{C} 10(S)$ (unnatural) & $156.9 \pm 32.7$ & $230.4 \pm 16.0$ \\
\hline TX64014 & $\mathrm{C} 4(S), \mathrm{C} 5(S), \mathrm{C} 10(R)$ (natural) & $16.4 \pm 2.3$ & $9.8 \pm 2.2$ \\
\hline TX285317 & $\mathrm{C} 4(R), \mathrm{C} 5(R), \mathrm{C} 10(S)$ (unnatural) & $347.0 \pm 35.0$ & $334.9 \pm 36.5$ \\
\hline
\end{tabular}

${ }^{a}$ Average values and standard deviations are derived from at least three experiments.

${ }^{\mathrm{b}} \mathrm{EC}_{2 \mathrm{x}}$ corresponds to the concentration of compound required to increase the reporter activity by 2 -fold relative to vehicle treatment.

${ }^{\mathrm{c}}$ Data from Reference [5]

\subsection{NRF2 is required for TX63541 antioxidant and anti-inflammatory activity}

The antioxidant and low-dose anti-inflammatory activities of the tpAIMs are dependent on NRF2 [4;36;37]. To evaluate whether NRF2 is also required for the antioxidant and anti-inflammatory activities of the sAIMs, we compared the effects of TX63541 to those of RTA 405 in RAW 264.7 mouse macrophage cells stimulated with interferon gamma (IFN $\gamma$ ). TX63541 and RTA 405 dose-dependently increased the mRNA levels of classic NRF2 target genes including: Nqo1, Gclm, and Txnrd1 (Fig. 4A-C). Both TX63541 and RTA 405 decreased IFN $\gamma$-induced expression of iNOS and MCP1 at the mRNA (Fig. 
5A,B) and the protein levels (Fig. 5C,D). In cells treated with RTA 405 or TX63541, we observed an increase in GCLM and a decrease in iNOS at similar concentrations (Fig. 5C), which is consistent with NRF2-mediated suppression of inflammation.

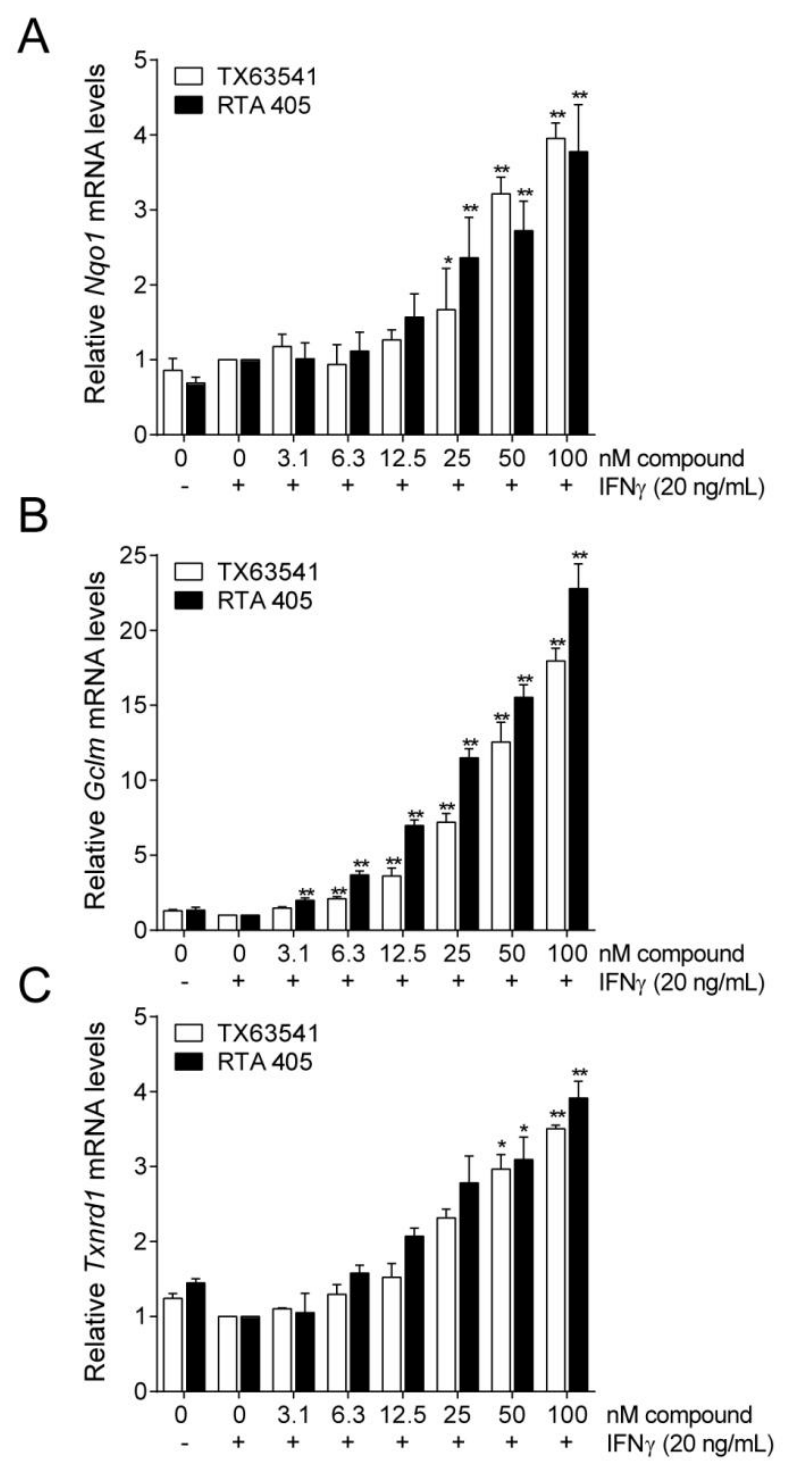

FIGURE 4. TX63541 and RTA 405 increase NRF2 target gene expression. A-C. RAW 264.7 macrophages were pretreated with the indicated concentrations of TX63541 or RTA 405 for $2 \mathrm{~h}$ and then treated with IFN $\gamma$ for an additional $24 \mathrm{~h}$. Message RNA levels of Nqol (A), Gclm (B), and Txnrdl (C) were measured by qPCR. Data are the average and SD of three independent experiments, shown as foldinduction relative to DMSO/IFN $\gamma$-treated samples, ${ }^{*} P<.05,{ }^{* *} P<.01$ compared to the corresponding $\mathrm{DMSO} / \mathrm{IFN} \gamma$-treated sample for each compound. 
A

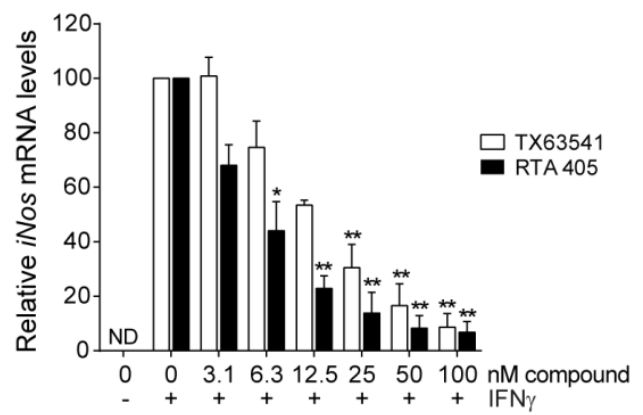

C

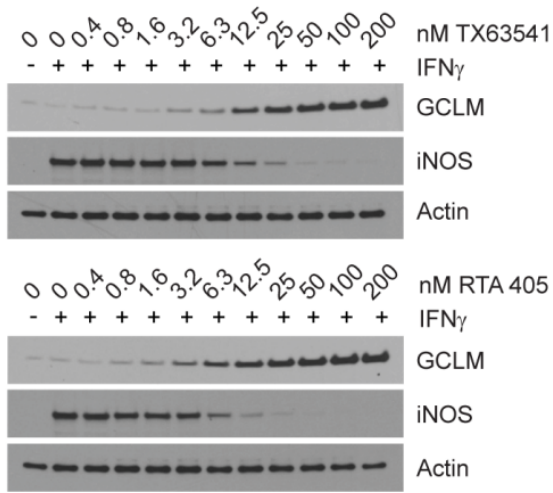

B
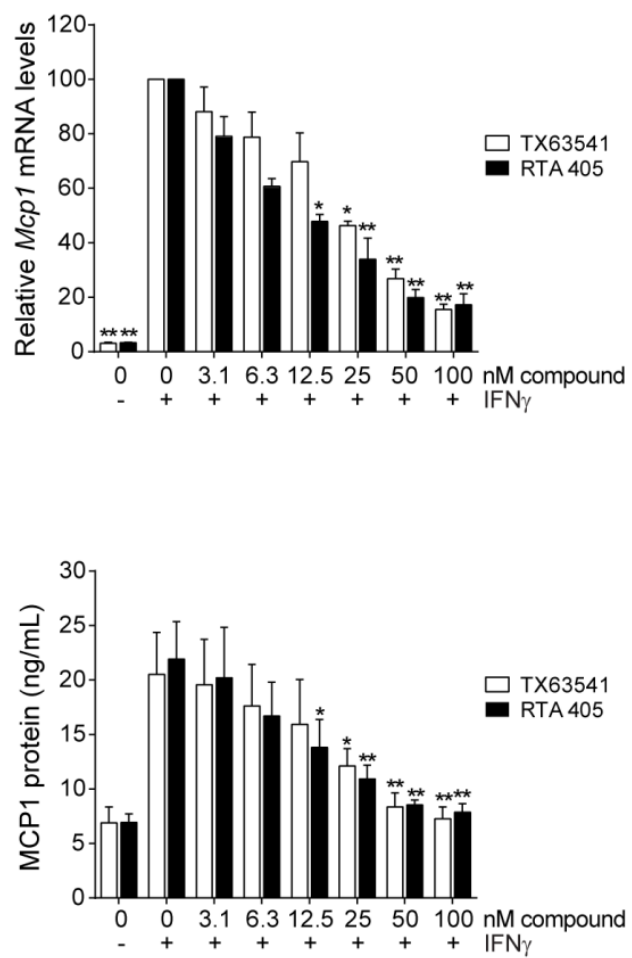

FIGURE 5. TX63541 and RTA 405 inhibit IFN $\gamma$-induced increases in iNOS and MCP1 levels. A-D. RAW 264.7 macrophages were pretreated with the indicated concentrations of TX63541 or RTA 405 for $2 \mathrm{~h}$ and then treated with IFN $\gamma$ for an additional $24 \mathrm{~h}$. A-B. iNos and Mcpl mRNA levels were measured by qPCR. Data are the average and SD of three independent experiments, shown as percentage of the DMSO/IFN $\gamma$-treated sample. C. GCLM and iNOS protein levels were assessed by western blot. Actin served as a loading control. Data are representative of two independent experiments. D. MCP1 protein concentrations in culture medium were measured by ELISA. Data are the average and SD of three independent experiments. $* P<.05, * * P<.01$ compared to DMSO/IFN $\gamma$-treated samples.

To evaluate whether NRF2 is required for sAIM-mediated suppression of pro-inflammatory mediators, we attempted to reduce NRF2 levels in RAW 264.7 cells using short interfering RNA (siRNA). Nrf2 mRNA levels were dramatically lower in cells that were transfected with Nrf2 siRNA than in cells that were mock-transfected or transfected with non-targeting siRNA (Fig. 6A,C). Neither TX63541 nor RTA 405 increased Nrf2 mRNA levels; however, both compounds increased nuclear NRF2 protein levels in mock-transfected and non-targeting siRNA-transfected cells (Fig. 6B,D). As expected, the TX63541- and RTA 405-mediated increase in nuclear NRF2 protein levels was strongly attenuated in Nrf2 siRNA-transfected cells (Fig. 6B,D). To determine whether low nuclear NRF2 levels correlated with 
reduced NRF2 activity, we assessed NRF2 target gene expression by qPCR. Compared with mock- and non-targeting siRNA-transfected cells, Nrf2 siRNA-transfected cells had slightly lower basal levels of Gclm and Txnrd1 mRNA (Fig. 6E,F). Treatment with TX63541 or RTA 405 increased Nqo1, Gclm, and Txnrdl expression in mock- and non-targeting siRNA-transfected cells, but not in Nrf2 siRNAtransfected cells (Fig. 6E,F). Taken together, these results demonstrate that Nrf2 siRNA reduced NRF2 activity in RAW 264.7 cells and that NRF2 is required for both tpAIMs and sAIMs to increase antioxidant gene expression.

To evaluate the dependence of tpAIMs and sAIMs on NRF2 for their anti-inflammatory activity, we next assessed NO, iNOS, and MCP-1 levels in the siRNA-transfected RAW 264.7 cells. Consistent with our observations in non-transfected cells (Fig. 3 and 5), TX63541 and RTA 405 inhibited IFN $\gamma$ induced NO (Fig. 7A), iNOS and MCP-1 mRNA (Fig. 7B,C) and iNOS and MCP-1 protein levels (Fig. 7D,E) in cells that were mock-transfected or transfected with non-targeting siRNA. In cells transfected with Nrf2 siRNA, the TX63541- and RTA 405-mediated suppression of NO, iNOS, and MCP-1 was significantly attenuated, although some suppression was still apparent at $100 \mathrm{nM}$. This could be due to incomplete knockdown of Nrf2, as demonstrated by low levels of nuclear NRF2 (Fig. 6B,D) and GCLM (Fig. 7D) in Nrf2 siRNA-transfected cells. We again noted that GCLM levels were increased by the same concentrations of RTA 405 and TX63541 that decreased iNOS levels, consistent with the dependence of tpAIMs on NRF2 for their anti-inflammatory activity at these doses [4;36;37]. At higher doses, tpAIMs have been shown to directly inhibit pro-inflammatory NF- $\kappa \mathrm{B}$ signaling by binding to $\mathrm{C} 179$ in IKK $\beta$ and inhibiting phosphorylation of I $\mathrm{B} \alpha$ [38;39]. To determine whether suppression of IFN $\gamma$-induced NO, iNOS, and MCP-1 could be attributed to a direct effect on the NF- $\mathrm{BB}$ pathway, we evaluated phosphoI $\mathrm{B} \alpha$ levels in RAW 264.7 cells treated with TX63541 or RTA 405. We found that treatment with IFN $\gamma$

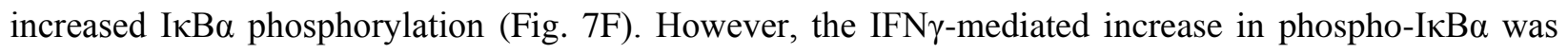
not reduced by TX63541 or RTA 405 at doses sufficient to suppress NO, iNOS, and MCP-1 levels. 
A

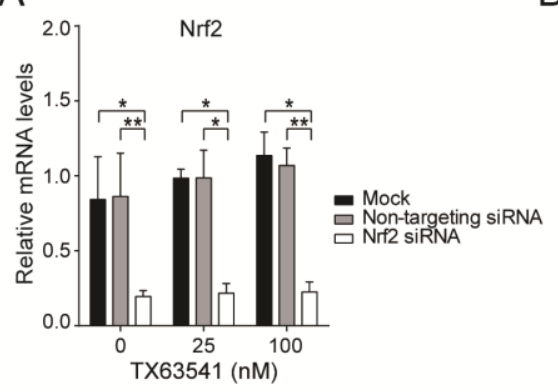

C

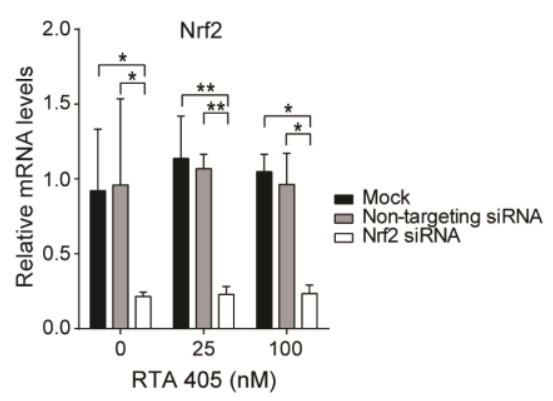

B
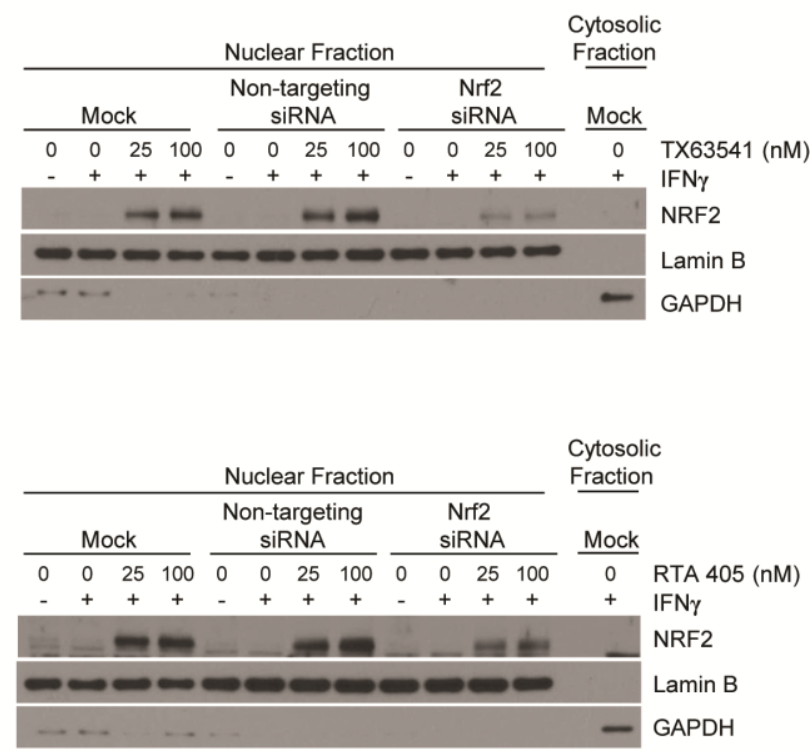

E
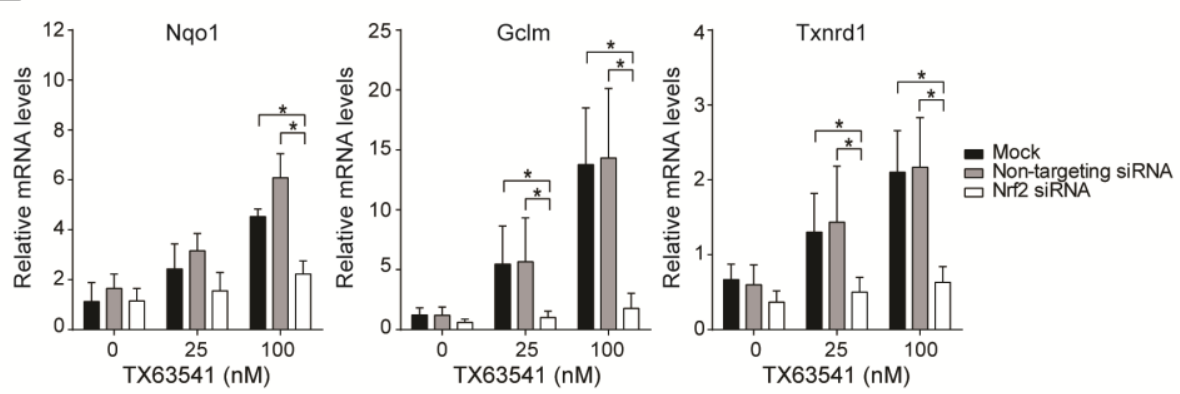

$\mathrm{F}$
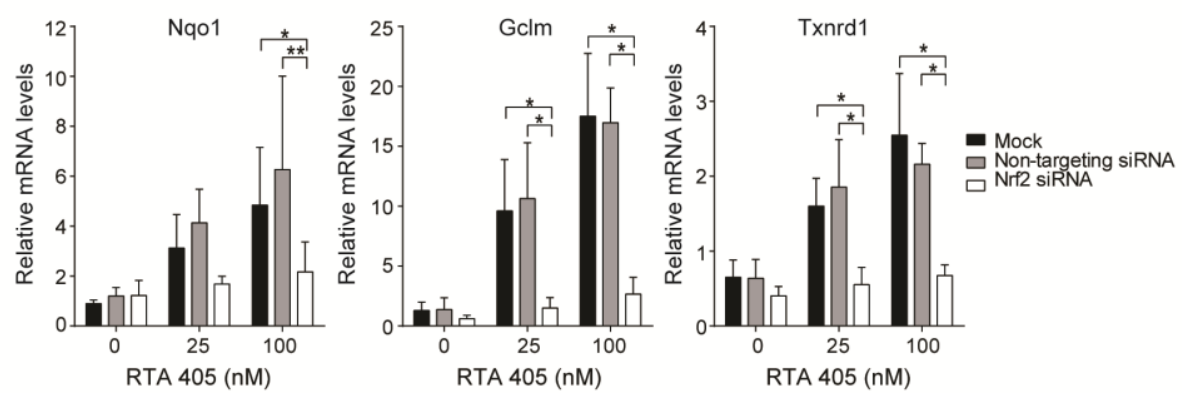

FIGURE 6. NRF2 is required for TX63541 and RTA405 to increase expression of Nqo1, Gclm, and Txnrd1. Following mock-transfection, or transfection with non-targeting or Nrf2 siRNA, cells were pretreated with the indicated concentrations of TX63541 (A,B,E) or RTA 405 (C,D,F) for $2 \mathrm{~h}$ and then treated with IFN $\gamma$ for an additional $24 \mathrm{~h}(\mathrm{~A}, \mathrm{C}, \mathrm{E}, \mathrm{F})$ or $2 \mathrm{~h}$ (B,D). A,C. Nrf2 mRNA levels were measured by qPCR. B,D. Nuclear NRF2 levels were assessed by western blot of fractionated cell lysates. Lamin B and GAPDH served as control nuclear and cytosolic proteins, respectively. Western blot data are representative of two independent experiments. E-F. Nqol, Gclm, and Txnrdl mRNA levels were measured by qPCR. All qPCR data are the average and SD of three independent experiments, shown as fold-induction relative to mock-transfected samples treated with DMSO alone. ${ }^{*} P<.05, * * P<.01$. 
A
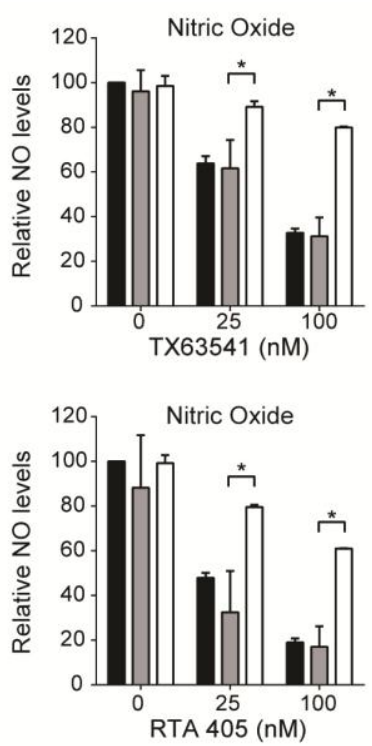

B
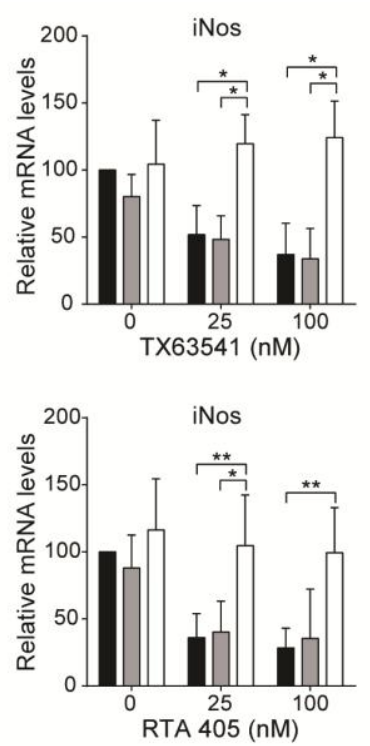
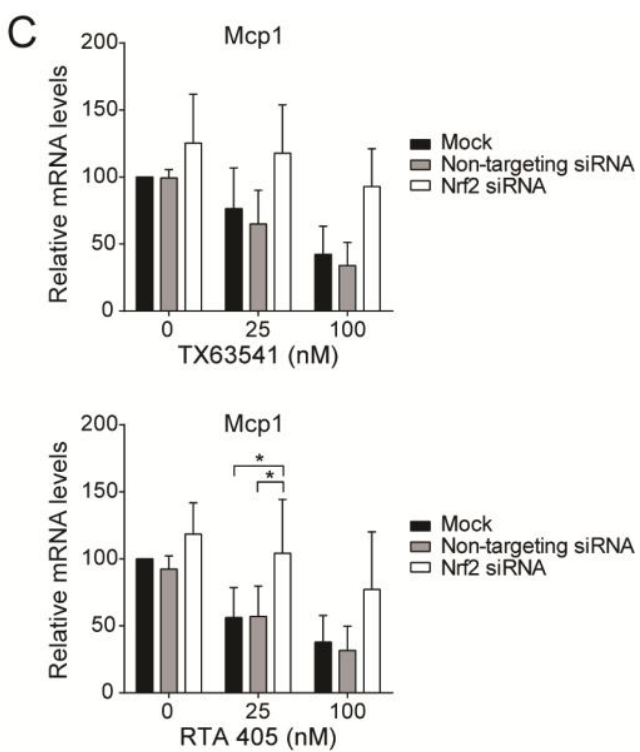

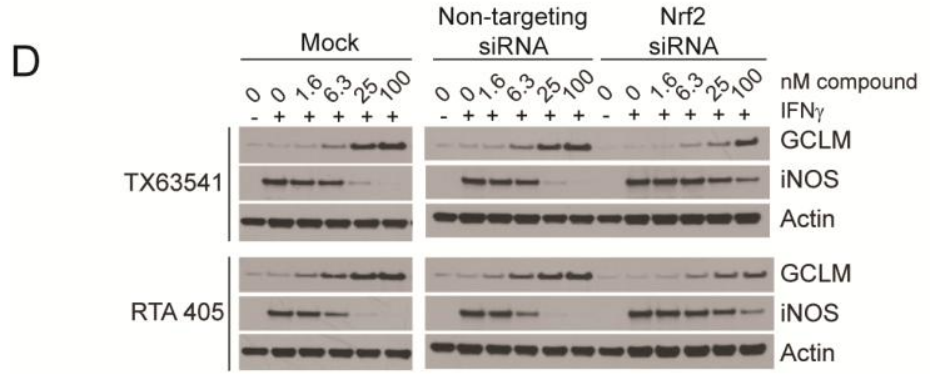

$\mathrm{E}$
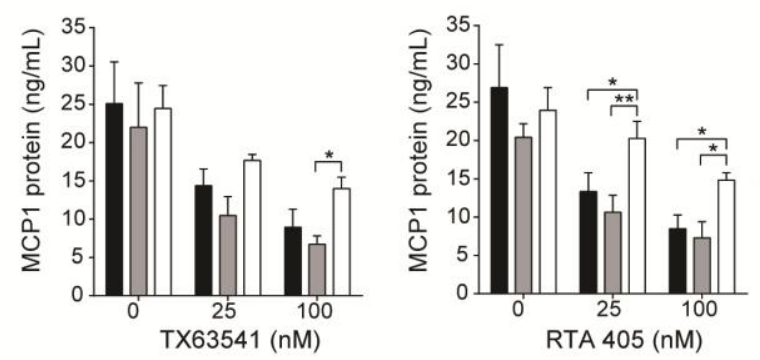

F

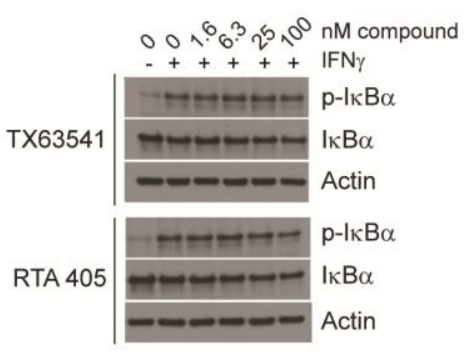

FIGURE 7. NRF2 is required for TX63541 and RTA405 to inhibit IFN $\gamma$-induced iNOS and MCP1 levels. Following transfection with siRNA (as in Figure 6), cells were pretreated with the indicated concentrations of TX63541 or RTA 405 for $2 \mathrm{~h}$ and then treated with IFN $\gamma$ for an additional $24 \mathrm{~h}$. A. Nitric oxide (NO) levels were measured in the media. B-C. iNOS and Mcpl mRNA levels were measured by qPCR. Data are the average and SD of three independent experiments, shown as percentage of the DMSO/IFN $\gamma$-treated mock-transfected sample. D. GCLM and iNOS protein levels were assessed by western blot. Actin served as a loading control. Data are representative of two independent experiments. E. MCP1 protein concentrations in culture medium were measured by ELISA. Data are the average and SD of three independent experiments. F. RAW 264.7 cells were pretreated with TX63541 or RTA 405 for

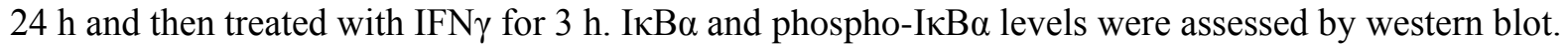
Actin served as a loading control. Data are representative of two independent experiments. $* P<.05$, ** $P$ $<.01$ compared to DMSO/IFN $\gamma$-treated samples. 


\subsection{KEAP1 C151 is required for TX63541 antioxidant activity}

The data presented above demonstrate that, like tpAIMs, the sAIM TX63541 has potent antioxidant and anti-inflammatory activity that is dependent on the presence of NRF2. To determine whether TX63541 increases NRF2 activity by binding to KEAP1 C151, we treated wild-type, KEAP1null, and KEAP1-C151S MEFs with TX63541 or RTA 405 and measured Nqo1 expression by qPCR. Both TX63541 and RTA 405 increased Nqol expression in a dose-dependent manner in wild-type MEFs (Fig. 8A,B). In contrast, Nqo1 expression was not increased in KEAP1-null or KEAP1-C151S MEFs following treatment with either compound. These data suggest that tpAIMs and sAIMs both bind to the C151 pocket in KEAP1.

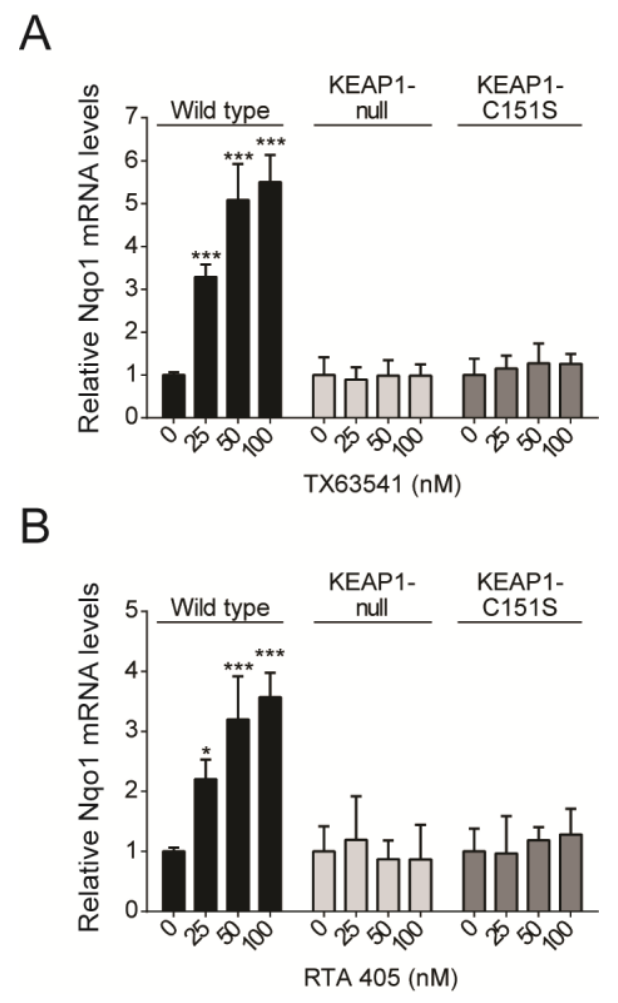

FIGURE 8. KEAP1 cysteine residue 151 is required for TX63541 and RTA 405 activity. A-B. Wildtype, KEAP1-null and KEAP1-C151S MEFs were treated with TX63541 (A) or RTA 405 (B) for $24 \mathrm{~h}$. Nqo1 mRNA levels were evaluated by qPCR and normalized to the DMSO-treated sample for each genotype (set to 1). Data are the average fold and range of three independent experiments. $* P<.05$, *** $P<.001$ compared to DMSO-treated samples. 


\subsection{Crystallization and structures of the KEAP1 BTB domain in complex with TX64014 or TX64063}

To better understand the stereoselectivity and explore the SAR of the sAIMs, we determined the crystal structure of the KEAP1 BTB domain (residues 49-182) in complex with TX64014 (pyrimidine sAIM) and TX64063 (pyrazole sAIM) (Fig. 2B and Table 2). When expressed alone, the BTB domain was prone to aggregation; however, co-expression and co-purification of the BTB domain with either one of the sAIMs improved protein solubility and purification characteristics, reduced protein aggregation, and enhanced crystallization.

The structures of the KEAP1 BTB domain in complex with TX64014 and TX64063 were determined at $2.61 \AA$ and $2.37 \AA$, respectively (Table 3). In agreement with previously described structures of BTB/POZ domains [40] and the structure of the KEAP1 BTB-CDDO complex [24], the KEAP1 BTB domain in complex with either one of the sAIMs crystallized as a domain-swapped homodimer. The crystals contained one monomer per asymmetric unit, with two monomers related by a 2-fold crystallographic symmetry along the $\alpha 1 / \alpha 2$ helices interface (Fig. 9A). Both KEAP1 BTB-sAIM complexes adopt a fold similar to other BTB/POZ domains [40] with three antiparallel $\beta$-strands enclosed by six $\alpha$-helices. The overall structures of the complexes are very similar to each other [Root mean square deviation (RMSD) of $0.36 \AA$ for equivalent $\mathrm{C} \alpha$ 's] (Fig. 9A) and to the structure of the BTB-CDDO complex (PDB ID 4CXT, RMSD of $0.33 \AA$ and $0.41 \AA$ for equivalent C $\alpha$ 's, (Fig. 9B,C). Minor structural differences between the complexes are observed for the loop connecting $\beta 1$ and $\beta 2$ strands (indicated by dashed arrow), which is positioned near the ligand binding site. The structural mobility of the loop is consistent with the general plasticity of the binding site. As expected, and in agreement with the structure of the BTB-CDDO complex, sAIM ligands are bound to C151 on the solvent exposed side of the BTB domain distal from the dimer interface. 
Table 3. Crystallographic statistics

\section{Data reduction}

\begin{tabular}{lcc}
\hline Compound & TX64014 & TX64063 \\
\hline Space group & $P 3_{1} 12$ & $P 3_{1} 12$ \\
Unit cell dimensions & & \\
$\quad a=b, c(\AA)$ & $43.2,135.2$ & $42.4,134.0$ \\
$\quad \alpha=\beta, \gamma\left({ }^{\circ}\right)$ & 90,120 & 90,120 \\
Resolution $(\AA)$ & $2.61(2.614-2.605)$ & $134-2.37(2.377-2.369)$ \\
Total number of reflections & $44180(411)$ & $55344(495)$ \\
Unique reflections & $4678(39)$ & $5900(50)$ \\
$R_{\text {merge }}(\%)^{\mathrm{a}}$ & $0.04(0.46)$ & $0.04(0.35)$ \\
$<\mathrm{I}>/ \sigma$ & $32.0(5.5)$ & $31.6(6.0)$ \\
Completeness $(\%)$ & $100(100)$ & $100(100)$ \\
Multiplicity & $9.4(10.5)$ & $9.4(9.9)$ \\
\hline
\end{tabular}

\section{Refinement}

Resolution $(\AA)$

Number of reflections

$R_{\text {work }}{ }^{\mathrm{b}} / R_{\text {free }}{ }^{\mathrm{c}}(\%)$

Mean B factor

Protein

Ligand

Water

Root mean square deviation

Bonds ( $\mathrm{A}$ )

Angles $\left({ }^{\circ}\right)$

Ramachandran Plot (\%)
$45.06-2.61$

4431

$20.4 / 24.5$

63.5

79.8

54.8

0.005

0.958

$97.5 / 2.5 / 0$
$44.68-2.37$

5591

$20.2 / 23.3$

63.0

79.8

65.7

0.007

1.297

94.0 / 6.0 / 0

\footnotetext{
${ }^{\mathrm{a}} R_{\text {merge }}=\sum_{h k l} \sum_{i}\left|I_{i}(h k l)-\langle I(h k l)\rangle\right| / \sum_{h k l} \sum_{i} I_{i}(h k l)$, where $I_{i}(h k l)$ is the intensity of the $i$ th measurement of reflection $h k l$ and $\langle I(h k l)\rangle$ is the average intensity of multiple observations of symmetry-related reflections of $I_{i}(h k l) .{ }^{\mathrm{b}} R_{\text {work }}=\sum_{h k l} \mid F_{o^{-}}$ $F_{c} \mid / \sum_{h k l} F_{o}$ ), where $F_{o}$ and $F_{c}$ are observed and calculated structure factors. ${ }^{c} R_{\text {free }}$ was calculated from a 5\% subset of reflections that were excluded from the refinement. Values in parentheses are for highest resolution shell.
} 

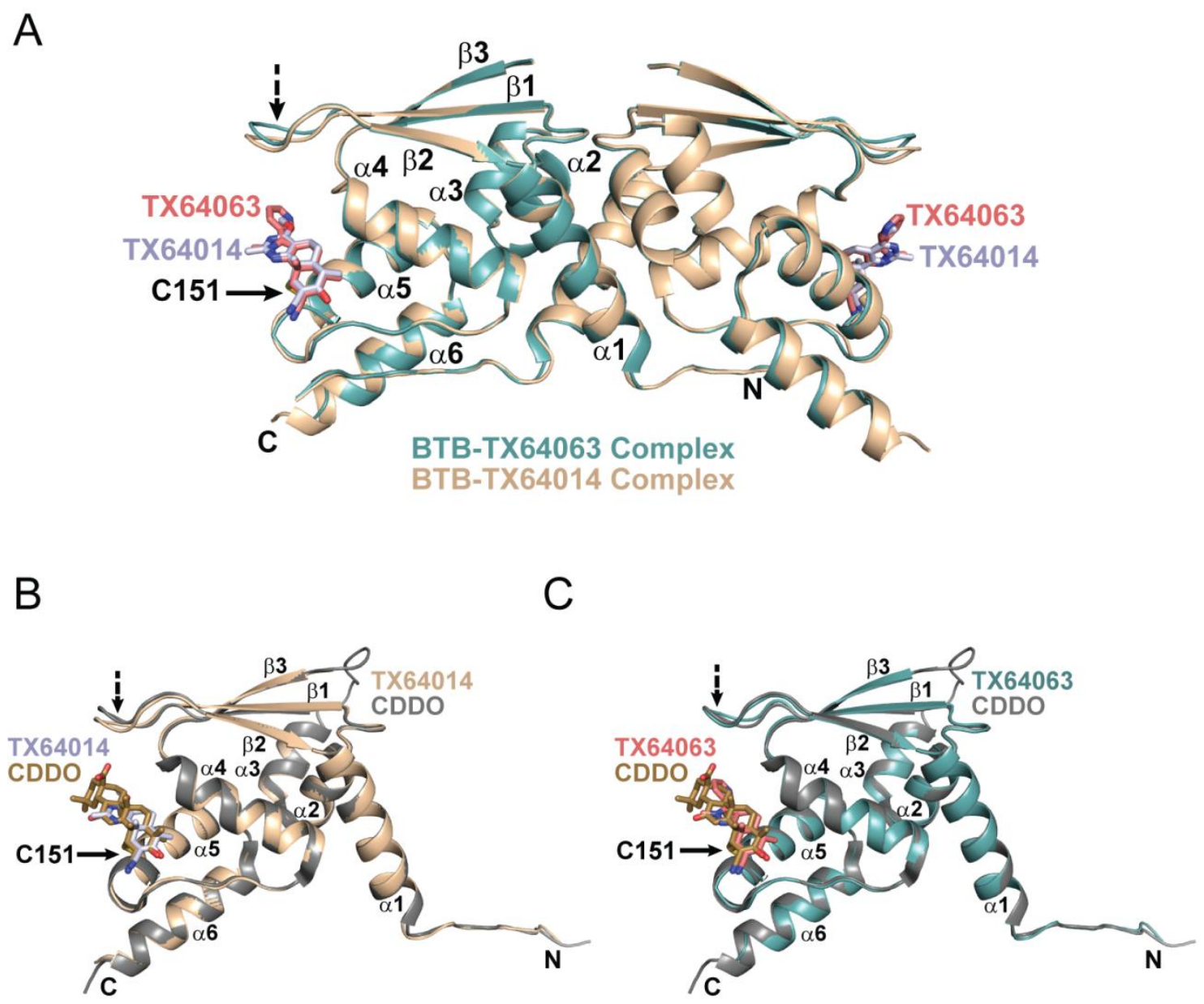

FIGURE 9. (A) Superposition of human KEAP1 BTB-sAIM complexes. Cartoon representation of the overall fold of the complexes as crystallographic dimers. Superposition of BTB-TX64063 complex (teal) to BTB-TX64014 complex (tan) with an RMSD of $0.36 \AA$ for equivalent C $\alpha$ 's. The N- and C-termini and main secondary structural elements are labeled on one monomer. Position of $\mathrm{C} 151$ is indicated by a black arrow. C151 and the ligands, TX64014 (light blue) and TX64063 (salmon), are shown as sticks. A flexible loop that showed structural motion between the complexes is indicated by a black dashed arrow. (B,C) Superposition of hKEAP1 BTB domain-CDDO complex to BTB-TX64014 (B) and BTB-TX64063 (C) complexes. The monomer complexes are very similar to each other with a RMSD of $0.33 \AA$ and 0.41 $\AA$ for equivalent $\mathrm{C} \alpha$, respectively. The black dashed arrows indicate the loop's structural motion between the CDDO and sAIM complexes. The loop connecting helix $\alpha 3$ to strand $\beta 3$ is disordered in the sAIM complexes but ordered in the CDDO complex. For all complexes, the compounds are on the solvent exposed C-terminal side of the BTB domain and form a Michael addition with C151. 
The electron density maps revealed that sAIMs were associated only with the C151 binding site and showed that both ligands were well ordered and present throughout the crystal lattice (Fig. 10). In both structures, a covalent bond was formed through a Michael addition between the C-1 carbon of the sAIM and the $\mathrm{S} \gamma$ of C151. This observation is consistent with the structure of the BTB-CDDO complex, and strongly supports the notion that sAIMs, like tpAIMs, activate NRF2 by covalent modification of KEAP1 C151. The electron density is most suggestive of the sAIM ligands being bound in enol form, as there was no indication of puckering along the C-1, C-2, or C-3 planes of the ligand's A-ring, and the C-3 enolic oxygen maintained a linear orientation within the same plane. The residues forming the sAIM binding site were also well represented by the density maps except for the sidechains of K131, E149 and $\mathrm{K} 150$, which lacked electron density beyond $\mathrm{C} \beta$.

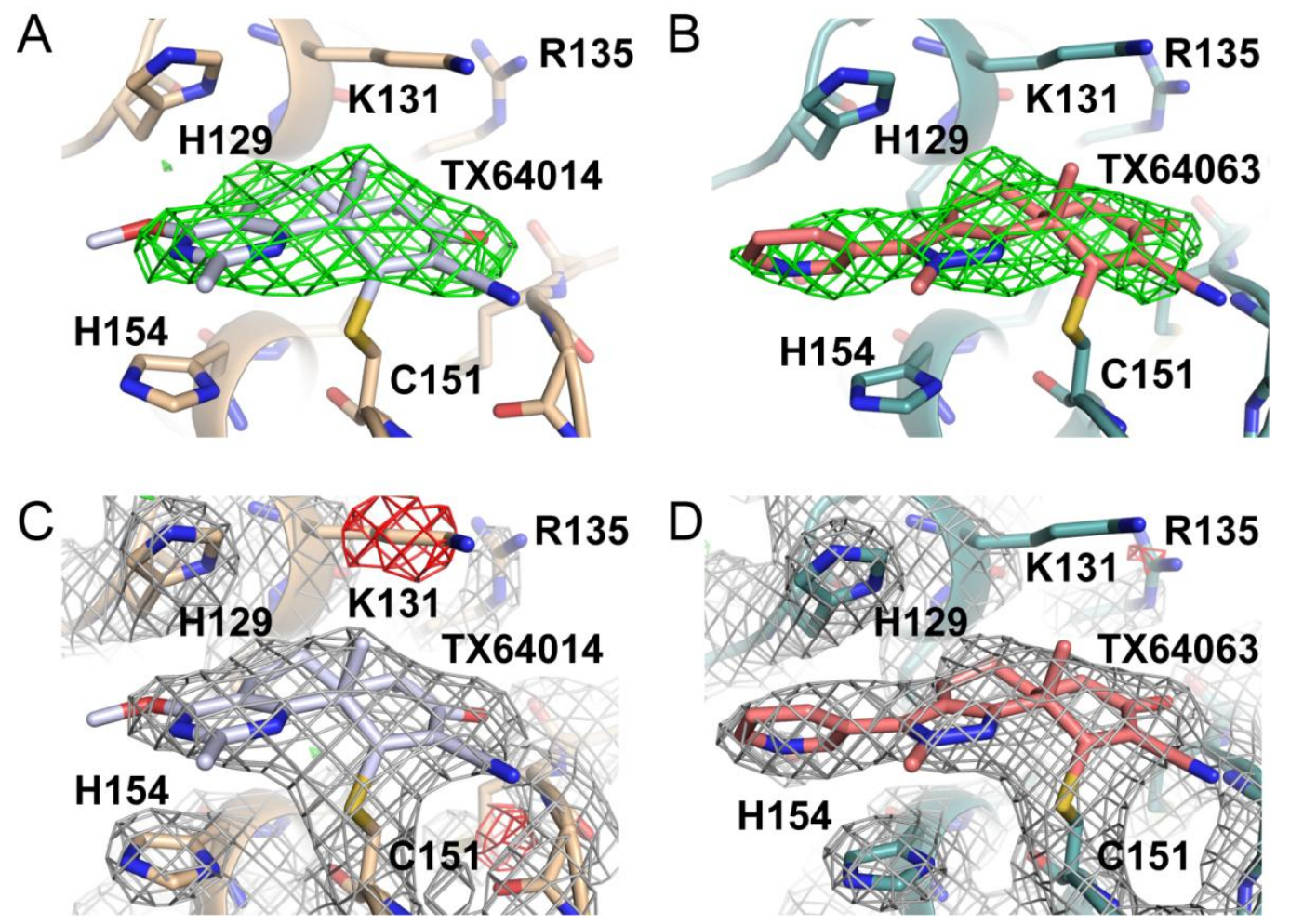

FIGURE 10. Electron density maps for the binding sites of the KEAP1 BTB-sAIM complexes. (A, B) OMIT electron density maps for TX64014 and TX64063. (C, D) Electron density maps for BTBTX64014 and BTB-TX64063 binding sites after final refinement. Densities are colored in gray for $2 F_{O}-$ $F_{C}$ map contoured at $1 \sigma$, and green or red for $F_{O}-F_{C}$ difference map contoured at $3 \sigma$ (positive and negative density, respectively). 
A detailed comparison of the binding modes of the pyrimidine and pyrazole sAIMs revealed a similar network of polar and hydrophobic interactions (Fig 11A,B). In both complexes, a $2.8 \AA$ hydrogen bond is formed between the C-3 enolic oxygen of each ligand and the G148 mainchain amide. The A and B rings of each ligand are covering a hydrophobic surface formed by V132, I145, M147, V155 and the aliphatic portion of K131. The G148 mainchain carbonyl is rotated inward in the TX64014 complex and forms hydrogen bonds with the mainchain amides of K150 (2.8 $⿱$ ) and C151 (2.9 $)$ ). In the TX64063 complex, the same carbonyl is rotated outward, presumably reflecting available protein dynamics around G148. Despite the different chemical nature of the C-ring, surprisingly, both sAIMs formed $\pi$-stacking interactions with $\mathrm{H} 129$ and H154, although the nature of the stacking interaction differs. In the structure with TX64014, the pyrimidine ring of the sAIM formed $\pi$-stacking interactions with H129 and H154; whereas in the structure with TX64063, the C-13-pyridyl ring formed $\pi$-stacking interactions with H129 and the pyrazole ring formed $\pi$-stacking interactions with H154 (Fig 11A,B and Fig 2). Both ligands also maintained Van der Waals interactions with the hydroxyl of Y85 via either the methyl of the TX64014 methoxy or the para-carbon on the pyridyl of TX64063 (Fig. 11A,B). Y85 is located on the flexible loop connecting the $\beta 1$ and $\beta 2$ strands (Fig. 9A), and may adjust its position in response to interaction with a particular ligand.

The electrostatic surface potential of the sAIM binding site (Fig. 11C,D) showed that C151 resides in a positively-charged valley formed primarily by the sidechains of residues H129, K131, R135, K150 and H154. The basic environment increases the nucleophilicity of the S $\gamma$ of C151 making it more reactive in the Michael addition to C-1 of the ligands [41]. The sAIM's conjugated ring system, as well as the C-14 methoxy of TX64014, leads to additional electrostatic interactions between the ligand and the surface of the protein (Fig. 11C,D). 

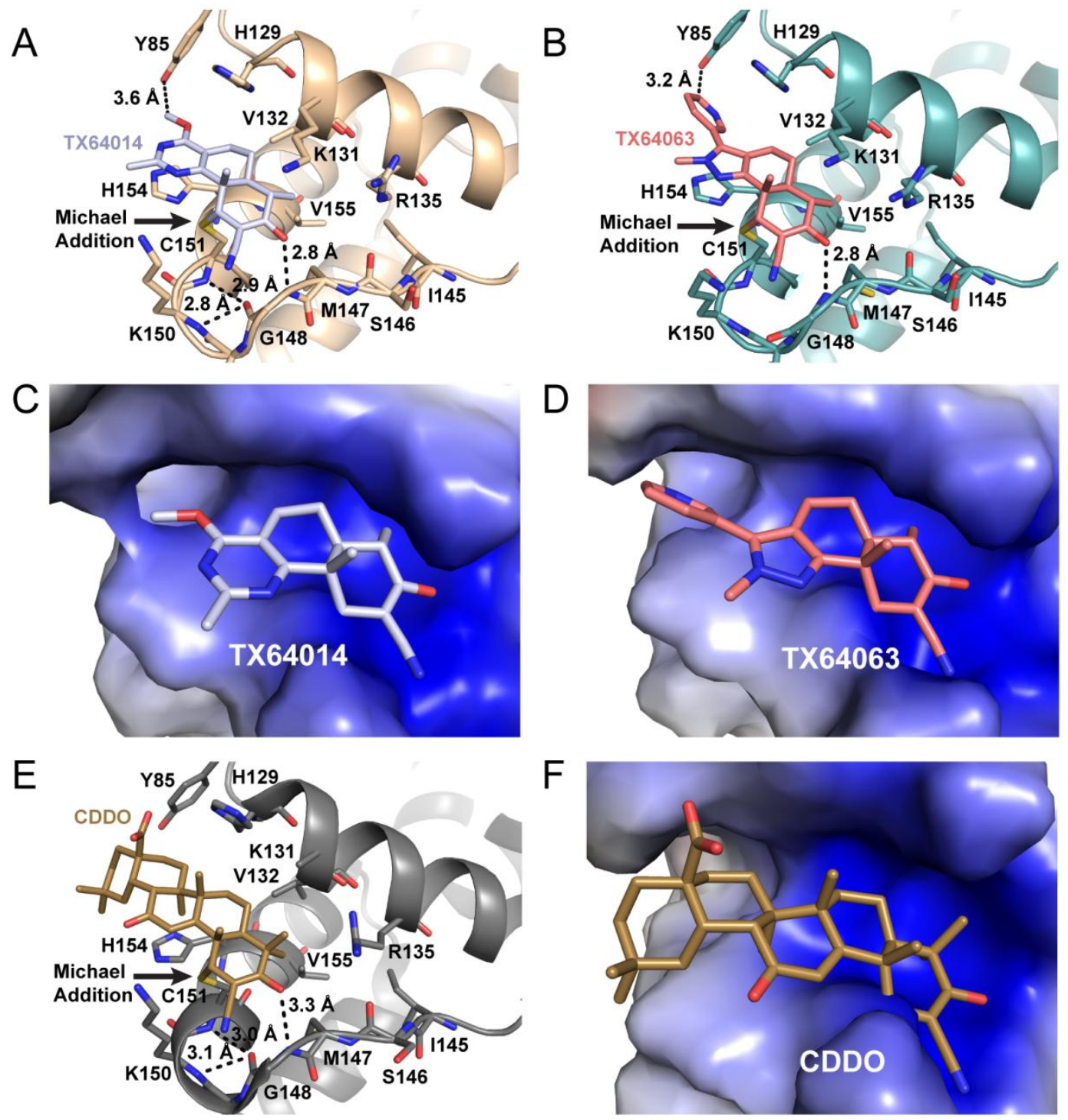

FIGURE 11. Comparison of the binding sites in the KEAP1 BTB-sAIM and KEAP1 BTB-CDDO complexes. The ligand binding sites of BTB-TX64014 complex (A), BTB-TX64063 complex (B), and BTB-CDDO complex (E) are shown separately in the same orientation. TX64014 (light blue), TX64063 (salmon), CDDO (brown) and residues of the ligand binding site are shown as sticks. The covalent bond formed by Michael addition between the ligands and C151 are indicated by a black arrow. C, D and F. Electrostatic surface potentials of the BTB-sAIM and BTB-CDDO complexes generated in the absence of TX64014 (C), TX64063 (D) and CDDO (F). K131 in the BTB-CDDO complex was modelled with PyMOL. Electrostatic surface potentials are color ramped from $-5 \mathrm{kT} / \mathrm{e}$ (red, negative) to $5 \mathrm{kT} / \mathrm{e}$ (blue, positive), with white being neutral. 
As described above, the natural enantiomer sAIMs exhibited potencies that are similar to those of tpAIMs (RTA 405) in the antioxidant and anti-inflammatory assays (Figs. 4-8 and Table 2), suggesting that sAIMs may mechanistically mimic tpAIMs via a similar KEAP1 binding mode. Indeed, when we compared the BTB-sAIM structures with the structure of the BTB-CDDO complex [24] we found that the composition of the sAIM binding site (Fig. 11A,B) was very similar to that of the CDDO binding site (Fig. 11E), with no additional residues involved in CDDO binding. Of note, although the BTB domain used in the CDDO complex contained a S172A mutation [24], the mutant structure is very similar to the structures of the wild-type BTB-sAIM complexes. The sAIM and tpAIM ligands were similarly oriented within the binding site. A hydrogen bond is formed between the C-3 enolic oxygen of each ligand and the G148 mainchain amide ( $3.3 \AA$ in the CDDO complex), and the A/B rings of each ligand maintained a similar net of hydrophobic interactions. In agreement with the observed flexibility of the G148 carbonyl, this carbonyl is rotated inward in the BTB-CDDO complex (similar to the BTB-TX64014 complex) and forms two slightly weaker hydrogen bonds with the mainchain amides of K150 (3.1 $)$ ) and C151 (3.0 ̊).

Differences between the interactions of the sAIMs and CDDO with the binding-site residues of KEAP1 begin at the C-ring, which may afford additional opportunities for sAIM optimization. The $\pi$ stacking and Van der Waals interactions formed by the sAIMs are absent in the BTB-CDDO complex. Instead, the C-, D- and E-rings of CDDO maintain hydrophobic and steric interactions with the sidechains of Y85, H129 and H154 (Fig. 11E). Moreover, compared to the BTB-sAIM complexes, there is minimal electrostatic complementation between ligand and protein surfaces in the BTB-CDDO complex, although potential complementation could come from the C-12 carbonyl and the C-17 carboxylate of CDDO (Fig. $11 F)$. Altogether, our comparative analysis of the sAIM and CDDO binding modes shows that the shape of the KEAP1 C151-containing binding site, as well as its mixed hydrophobic and polar nature, allows for specific, yet adaptable interactions with both types of ligands, and may explain the similar potency of these compounds in biological assays. 


\section{Discussion}

The results from this study demonstrate that purely synthetic pyrimidine and pyrazole sAIMs are potent antioxidant and anti-inflammatory compounds that closely mimic the activity of semi-synthetic oleanolic acid-derived tpAIMs. We have generated sAIMs with stereoselective KEAP1 C151-binding by maintaining the A-ring cyanoenone Michael acceptor, the transfused bicyclic [6.6] ring system, and the apical methyl group at C-10. Introduction of the fused heterocyclic C-ring allowed further expansion of the SAR as well as manipulation of the physicochemical properties essential for drug design.

In all of the biological assays, sAIMs performed similarly to tpAIMs. RTA 405 and TX63541 increased NRF2 activity at similar concentrations, as evidenced by ARE-luciferase reporter activity and qPCR of NRF2 target genes in RAW 264.7 macrophages and murine embryonic fibroblasts. Both RTA 405 and TX63541 also exhibited similar potency toward suppression of several markers of inflammation, including nitric oxide, iNOS, and MCP-1, by a mechanism that required NRF2. It is well-known that both iNOS and MCP-1 are targets of NF-kB. Since higher concentrations of tpAIMs have been shown to inhibit the NF- $\kappa$ B pathway by binding to IKK $\beta$ and inhibiting its kinase activity [5;38;39], this raised the formal possibility direct inhibition of IKK $\beta$ could contribute to suppression of these inflammatory

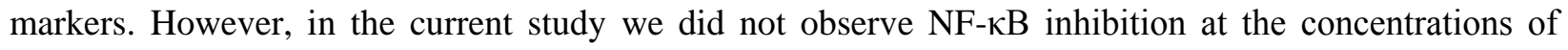
tpAIMs and sAIMs that effectively suppressed markers of inflammation and increased NRF2 activity. These findings are consistent with the results of another study, which found the anti-inflammatory activity of lower concentrations of the tpAIM dh404 to be dependent on NRF2, but independent of NF- $\mathrm{KB}$ [37]. NRF2 is known to suppress the expression of pro-inflammatory genes [42] and the lower-concentration anti-inflammatory activity of the tpAIMs has previously been shown to be NRF2-dependent [4;36]. Moreover, Kobayashi and colleagues recently identified NRF2 as an upstream regulator of cytokine production and demonstrated that NRF2 binds to DNA in the proximity of pro-inflammatory genes and inhibits recruitment of RNA polymerase II [43]. Therefore, although the precise molecular mechanism 
warrants further investigation, NRF2 activation by tpAIMs and sAIMs appears to directly inhibit expression of pro-inflammatory mediators.

The tpAIMs are highly reversible Michael acceptors capable of rapid and fleeting associations with thiols such as those in reduced glutathione and dithiothreitol [18]. The importance of KEAP1 C151 in mediating NRF2 activation by the tpAIMs CDDO-Im and dh404 has been demonstrated previously $[20 ; 22 ; 23]$. In the present study, we found that $\mathrm{C} 151$ is required for the interaction between KEAP1 and a biotinylated tpAIM probe and for NRF2 activation by a prototypic tpAIM, RTA 405. Consistent with these findings, Cleasby et al. recently solved the crystal structure of the human KEAP1 BTB domain in complex with CDDO and showed that this tpAIM forms a covalent bond with C151 through a Michael addition reaction [24]. Our own structural data, presented here, demonstrate a covalent interaction between the sAIMs and KEAP1 C151. In addition, like RTA 405, the sAIM TX63541 did not increase NRF2 activity in KEAP1-C151 mutant cells, which further underscores the importance of this residue in AIM-mediated NRF2 activation. KEAP1 C151 is modified by several reactive molecules and cytoprotective compounds, such as tBHQ, DMF, and sulforaphane $[21 ; 44]$. However, these compounds induce NRF2 much more weakly than the tpAIMs and sAIMs do, suggesting that oxidation or alkylation of $\mathrm{C} 151$ alone does not result in optimal NRF2 activation. The additional molecular interactions that we and others [24] have observed between the AIMs and the residues that line the KEAP1 C151 binding pocket likely contribute to their potency and target selectivity.

With only one absolute stereochemical configuration available in oleanolic acid, the natural product from which the tpAIMs are derived, it was not possible to assess antipodal drug recognition in the tpAIMs - the hallmark of stereospecific drug/protein interaction $[45 ; 46]$. Synthesis of the sAIMs provided the opportunity to assess the stereoselectivity of the sAIMs and to further our understanding of the tpAIM SAR. The dramatic loss of potency that we observed for sAIMs with an unnatural configuration $(\mathrm{C}-4(R), \mathrm{C}-5(R), \mathrm{C}-10(S))$, as compared to those with a natural configuration $(\mathrm{C}-4(S), \mathrm{C}$ $5(S), \mathrm{C}-10(R))$, supports the presence of a stereoselective binding pocket centered around KEAP1 C151. Notably, the electrophilic nature of the cyanoenone Michael acceptor is the same in each of the sAIM 
enantiomer pairs, which suggests that the potency of the sAIMs is not due to non-specific interactions with reactive cysteine residues. As mentioned previously, the natural sAIMs have the same configuration as the tpAIMs, which implies that the exquisite potency of the tpAIMs toward activation of the KEAP1/NRF2 pathway is also largely due to stereospecific recognition of this class of ligands by KEAP1. The apical C-10 methyl group plays a critical role in this recognition as it ensures facial selectivity in the addition of $\mathrm{C} 151$ to the si face of the Michael acceptor. The substantially less potent NRF2 activation by the unnatural sAIMs may be due to fewer favorable interactions at the C151 binding site; however, the notion that the activity of the unnatural enantiomers is mediated by ligation of another sensor cysteine residue on KEAP1 cannot be excluded [47;48].

Having clearly established the foundation for stereoselective recognition of the sAIMs by KEAP1, we sought a more detailed view of the protein/ligand interaction at the molecular level through high resolution X-ray diffraction. The structures of human KEAP1 BTB domain in complex with two different sAIMs, TX64014 and TX64063, provide valuable insights regarding key interactions that drive the potency and selectivity of the sAIMs and are consistent with stereoselective binding of sAIMs to KEAP1 C151. The mixed hydrophobic and polar nature of the KEAP1 C151 binding site allows for specific yet adaptable interactions that are influenced by the chemical properties of the ligand. It is tempting to speculate that KEAP1 evolved such flexibility at the C151 binding site in order to allow different "stress mediators" to engage the cytoprotective KEAP1/NRF2 system. Through the modified Cring, sAIMs form interactions with residues in the KEAP1 binding site that are distinct from the interactions formed by the tpAIMs, but still result in similar potencies in cell-based biological assays. The structural and chemical properties of the sAIMs and tpAIMs may allow the ligands to come in close proximity to the $\mathrm{C} 151$-containing binding site. Proper alignment might then facilitate stereospecific nucleophilic addition by $\mathrm{C} 151$ to the si face of the cyanoenone, leading to a very acidic enol that is further stabilized through a hydrogen bond to the protein mainchain. Although the mechanism of sAIM-mediated NRF2 stabilization cannot be determined from the static picture provided by the KEAP1 BTB structure alone, a "conformation cycling" model has been proposed for other C151-dependent inducers, in which 
binding to C151 results in a conformational change in KEAP1 that prevents ubiquitination of NRF2 [49;50]. Equipped with this understanding, medicinal chemistry has a rational basis for further sAIM optimization focused on improving both drug potency and physicochemical properties; the essential components for pharmacological modulation of a desired drug target in vivo.

Despite the recent clinical success of a cysteine-modifying compound, Tecfidera (BG-12, DMF) [51], "non-traditional" drug development using Michael acceptors and other electrophilic cysteinemodifying agents has lagged due to anticipation of off-target and idiosyncratic toxicities [52]. Nevertheless, there has been a recent resurgence of interest in the development of irreversible covalent protein kinase inhibitors (PKIs) by targeting neighboring cysteine residues in adenosine triphosphate (ATP) catalytic sites with Michael acceptors linked to ATP mimetics [53]. In contrast to the irreversible adducts that are formed between thiols and these PKIs, the adducts formed between thiols and tpAIMs are highly-reversible [18]. Both the reversibility of the Michael acceptor and the strict stereochemical bias-for the desired target protein are essential to the tpAIM mechanism of action. The transient association of tpAIMs with thiols, in concert with their stereoselectivity toward the local molecular environment proximal to KEAP1 C151 may contribute to the tolerability profiles of bardoxolone methyl that have been observed during clinical development [7-9;54-56].

\section{Conclusions}

In the current report, we have established and validated the sAIMs as informative tpAIM mimetics. We have demonstrated that they bind to C151 in the KEAP1 BTB domain and interact in a stereospecific manner with additional residues comprising the binding pocket, which ultimately results in NRF2 nuclear translocation and engagement of target AREs. Key molecular features governing this process include an accessible low pKa thiol, a highly reactive cysteine-specific Michael acceptor (favoring a high dissociation rate), and significant molecular recognition between protein and ligand. To date, this approach has found clinical utility only with respect to KEAP1, with long term clinical safety 
still being evaluated. Nonetheless, as we gain further understanding of how redox sensitive cysteine residues control post-translational modification of proteins at the molecular level, this approach holds great promise as an innovative method for developing novel therapeutics in numerous chronic disease indications. 


\begin{abstract}
Abbreviations:
The abbreviations used are: ANOVA, analysis of variance; BME, $\beta$-mercaptoethanol; BTB, Broadcomplex, Tramtrack and Bric-a-brac; DEM, diethyl maleate; DMF, dimethyl fumarate; Kan, Kanamycin; MEF, murine embryonic fibroblast; PDB, Protein Data Bank; PKI, protein kinase inhibitor; RMSD, root mean square deviation; sAIM, synthetic antioxidant inflammation modulator; SAR, structure-activity relationship; tBHQ, tert-butylhydroquinone; tpAIM, triterpenoid antioxidant inflammation modulator.
\end{abstract}

Acknowledgements: We thank Zohre German and Linda Hannigan for expert assistance with molecular biology and cell culture. We also thank Martha Hotema for the chiral HPLC isolation of TX64014, TX64063 and TX285317. For crystal structure analysis, diffraction data were collected at beamline 17-ID in the facilities of the Industrial Macromolecular Crystallography Association Collaborative Access Team (IMCA-CAT) at the Advanced Photon Source. Use of the IMCA-CAT beamline was supported by the companies of the Industrial Macromolecular Crystallography Association through a contract with Hauptman-Woodward Medical Research Institute. Use of the Advance Proton Source was supported by the U.S. Department of Energy, Office of Science, Office of Basic Energy Sciences, under Contract No. DE-AC02-06CH11357. 
Reference List

1. Stewart,J.D., Hengstler,J.G., and Bolt,H.M. (2011) Control of oxidative stress by the Keap1-Nrf2 pathway. Arch.Toxicol., 85, 239.

2. Suzuki,T., Motohashi,H., and Yamamoto,M. (2013) Toward clinical application of the Keap1Nrf2 pathway. Trends Pharmacol.Sci., 34, 340-346.

3. Kim,J., Cha,Y.N., and Surh,Y.J. (2010) A protective role of nuclear factor-erythroid 2-related factor-2 (Nrf2) in inflammatory disorders. Mutat.Res., 690, 12-23.

4. Dinkova-Kostova,A.T., Liby,K.T., Stephenson,K.K., Holtzclaw,W.D., Gao,X., Suh,N., Williams,C., Risingsong,R., Honda,T., Gribble,G.W., Sporn,M.B., and Talalay,P. (2005) Extremely potent triterpenoid inducers of the phase 2 response: correlations of protection against oxidant and inflammatory stress. Proc.Natl.Acad.Sci.U.S.A, 102, 4584-4589.

5. Probst,B.L., Trevino,I., McCauley,L., Bumeister,R., Dulubova,I., Wigley,W.C., and Ferguson,D.A. (2015) RTA 408, A Novel Synthetic Triterpenoid with Broad Anticancer and Anti-Inflammatory Activity. PLoS.ONE., 10, e0122942.

6. Liby,K.T. and Sporn,M.B. (2012) Synthetic oleanane triterpenoids: multifunctional drugs with a broad range of applications for prevention and treatment of chronic disease. Pharmacol.Rev., 64, 972-1003.

7. Hong,D.S., Kurzrock,R., Supko,J.G., He,X., Naing,A., Wheler,J., Lawrence,D., Eder,J.P., Meyer,C.J., Ferguson,D.A., Mier,J., Konopleva,M., Konoplev,S., Andreeff,M., Kufe,D., Lazarus,H., Shapiro,G.I., and Dezube,B.J. (2012) A Phase I First-in-Human Trial of Bardoxolone Methyl in Patients with Advanced Solid Tumors and Lymphomas. Clin.Cancer Res..

8. Pergola,P., Krauth,M., Huff,W., Ferguson,D., Ruiz,S., Meyer,C., and Warnock,D.G. (2011) Effect of Bardoxolone on Kidney Function in Patients with T2D and Stage 3b - 4 CKD. Am J Nephrol., 33, 469-476.

9. Pergola,P.E., Raskin,P., Toto,R.D., Meyer,C.J., Huff,J.W., Grossman,E.B., Krauth,M., Ruiz,S., Audhya,P., Christ-Schmidt,H., Wittes,J., and Warnock,D.G. (2011) Bardoxolone methyl and kidney function in CKD with type 2 diabetes. N.Engl.J Med., 365, 327-336.

10. Reata Pharmaceuticals (2015) Bardoxolone Methyl Evaluation in Patients With Pulmonary Arterial Hypertension (PAH) - LARIAT. ClinicalTrials.gov [Internet]. National Library of Medicine (US). 2000- [cited 2016 Jun 09]. Available from: http://ClinicalTrials.gov/show/NCT02036970 NLM Identifier: NCT02036970, Bethesda (MD).

11. Reata Pharmaceuticals,I. (2016) Bardoxolone Methyl in Patients With Connective Tissue Disease-associated Pulmonary Arterial Hypertension - CATALYST. ClinicalTrials.gov [Internet]. National Library of Medicine (US). 2000- [cited 2016 Feb 20]. Available from: http://ClinicalTrials.gov/show/NCT02657356 NLM Identifier: NCT02657356, Bethesda, MD.

12. Kyowa Hakko Kirin Company,L. (2015) RTA 402 Phase 2 Clinical Trial (A Randomized, Double-blind, Placebo-controlled Clinical Trial in Patients With Chronic Kidney Disease and Type 2 Diabetes). ClinicalTrial.gov [Internet]. National Library of Medicine (US). 2000- [cited 
2016 Jun 09]. Available from: http://ClinicalTrials.gov/show/NCT02316821 NLM Identifier: NCT02316821, Bethesda (MD).

13. Reata Pharmaceuticals and AbbVie (2016) RTA 408 Capsules in Patients With Friedreich's Ataxia - MOXIe. ClinicalTrials.gov [Internet]. National Library of Medicine (US). 2000- [cited 2016 Jun 09]. Available from: http://clinicaltrials.gov/show/NCT02255435 NLM Identifier: NCT02255435, Bethesda (MD).

14. Reata Pharmaceuticals and AbbVie (2015) RTA 408 Capsules in Patients With Mitochondrial Myopathy - MOTOR. ClinicalTrial.gov [Internet]. National Library of Medicine (US). 2000[cited 2016 Jun 09]. Available from: http://ClinicalTrials.gov/show/NCT02255422 NLM Identifier: NCT02255422, Bethesda (MD).

15. Reata Pharmaceuticals and AbbVie (2015) RTA 408 Ophthalmic Suspension for the Prevention of Corneal Endothelial Cell Loss Following Cataract Surgery - GUARD. ClinicalTrials.gov [Internet]. National Library of Medicine (US). 2000- [cited 2016 Jun 09]. Available from: http://ClinicalTrials.gov/show/NCT02128113 NLM Identifier: NCT02128133, Bethesda (MD).

16. Reata Pharmaceuticals,I. and AbbVie (2016) RTA 408 Capsules in Patients with Melanoma REVEAL. ClinicalTrial.gov [Internet]. National Library of Medicine (US). 2000- [cited 2016 Jun 09]. Available from: http://ClinicalTrials.gov/show/NCT02259231 NLM Identifier: NCT02259231, Bethesda (MD).

17. Honda,T., Rounds,B.V., Bore,L., Finlay,H.J., Favaloro,F.G., Jr., Suh,N., Wang,Y., Sporn,M.B., and Gribble,G.W. (2000) Synthetic oleanane and ursane triterpenoids with modified rings A and C: a series of highly active inhibitors of nitric oxide production in mouse macrophages. J.Med.Chem., 43, 4233-4246.

18. Couch,R.D., Browning,R.G., Honda,T., Gribble,G.W., Wright,D.L., Sporn,M.B., and Anderson,A.C. (2005) Studies on the reactivity of CDDO, a promising new chemopreventive and chemotherapeutic agent: implications for a molecular mechanism of action. Bioorg.Med.Chem.Lett., 15, 2215-2219.

19. Yamamoto,T., Suzuki,T., Kobayashi,A., Wakabayashi,J., Maher,J., Motohashi,H., and Yamamoto,M. (2008) Physiological significance of reactive cysteine residues of Keap1 in determining Nrf2 activity. Mol.Cell Biol., 28, 2758-2770.

20. Saito,R., Suzuki,T., Hiramoto,K., Asami,S., Naganuma,E., Suda,H., Iso,T., Yamamoto,H., Morita,M., Baird,L., Furusawa,Y., Negishi,T., Ichinose,M., and Yamamoto,M. (2015) Characterizations of Three Major Cysteine Sensors of Keap1 in Stress Response. Mol.Cell Biol., 36, 271-284.

21. Takaya,K., Suzuki,T., Motohashi,H., Onodera,K., Satomi,S., Kensler,T.W., and Yamamoto,M. (2012) Validation of the multiple sensor mechanism of the Keap1-Nrf2 system. Free Radic.Biol.Med., 53, 817-827.

22. Ichikawa,T., Li,J., Meyer,C.J., Janicki,J.S., Hannink,M., and Cui,T. (2009) Dihydro-CDDOtrifluoroethyl amide (dh404), a novel Nrf2 activator, suppresses oxidative stress in cardiomyocytes. PLoS.ONE., 4, e8391. 
23. Eggler,A.L., Small,E., Hannink,M., and Mesecar,A.D. (2009) Cul3-mediated Nrf2 ubiquitination and antioxidant response element (ARE) activation are dependent on the partial molar volume at position 151 of Keap1. Biochem.J, 422, 171-180.

24. Cleasby,A., Yon,J., Day,P.J., Richardson,C., Tickle,I.J., Williams,P.A., Callahan,J.F., Carr,R., Concha,N., Kerns,J.K., Qi,H., Sweitzer,T., Ward,P., and Davies,T.G. (2014) Structure of the BTB Domain of Keap1 and Its Interaction with the Triterpenoid Antagonist CDDO. PLoS.ONE., 9, e98896.

25. Honda,T., Janosik,T., Honda,Y., Han,J., Liby,K.T., Williams,C.R., Couch,R.D., Anderson,A.C., Sporn,M.B., and Gribble,G.W. (2004) Design, synthesis, and biological evaluation of biotin conjugates of 2-cyano-3,12-dioxooleana-1,9(11)-dien-28-oic acid for the isolation of the protein targets. J.Med.Chem., 47, 4923-4932.

26. Ohta,T., Iijima,K., Miyamoto,M., Nakahara,I., Tanaka,H., Ohtsuji,M., Suzuki,T., Kobayashi,A., Yokota,J., Sakiyama,T., Shibata,T., Yamamoto,M., and Hirohashi,S. (2008) Loss of Keap1 function activates Nrf2 and provides advantages for lung cancer cell growth. Cancer Res., 68, 1303-1309.

27. Livak,K.J. and Schmittgen,T.D. (2001) Analysis of relative gene expression data using real-time quantitative PCR and the 2(-Delta Delta C(T)) Method. Methods, 25, 402-408.

28. McCoy,A.J., Grosse-Kunstleve,R.W., Adams,P.D., Winn,M.D., Storoni,L.C., and Read,R.J. (2007) Phaser crystallographic software. J.Appl.Crystallogr., 40, 658-674.

29. Murshudov,G.N., Skubak,P., Lebedev,A.A., Pannu,N.S., Steiner,R.A., Nicholls,R.A., Winn,M.D., Long,F., and Vagin,A.A. (2011) REFMAC5 for the refinement of macromolecular crystal structures. Acta Crystallogr.D.Biol.Crystallogr., 67, 355-367.

30. Emsley,P., Lohkamp,B., Scott,W.G., and Cowtan,K. (2010) Features and development of Coot. Acta Crystallogr.D.Biol.Crystallogr., 66, 486-501.

31. Lebedev,A.A., Young,P., Isupov,M.N., Moroz,O.V., Vagin,A.A., and Murshudov,G.N. (2012) JLigand: a graphical tool for the CCP4 template-restraint library. Acta Crystallogr.D.Biol.Crystallogr., 68, 431-440.

32. Winn,M.D., Ballard,C.C., Cowtan,K.D., Dodson,E.J., Emsley,P., Evans,P.R., Keegan,R.M., Krissinel,E.B., Leslie,A.G., McCoy,A., McNicholas,S.J., Murshudov,G.N., Pannu,N.S., Potterton,E.A., Powell,H.R., Read,R.J., Vagin,A., and Wilson,K.S. (2011) Overview of the CCP4 suite and current developments. Acta Crystallogr.D.Biol.Crystallogr., 67, 235-242.

33. Honda,T., Favaloro,F.G., Jr., Janosik,T., Honda,Y., Suh,N., Sporn,M.B., and Gribble,G.W. (2003) Efficient synthesis of (-)- and (+)-tricyclic compounds with enone functionalities in rings A and C. A novel class of orally active anti-inflammatory and cancer chemopreventive agents. Org.Biomol.Chem., 1, 4384-4391.

34. Liby,K., Royce,D.B., Williams,C.R., Risingsong,R., Yore,M.M., Honda,T., Gribble,G.W., Dmitrovsky,E., Sporn,T.A., and Sporn,M.B. (2007) The synthetic triterpenoids CDDO-methyl ester and CDDO-ethyl amide prevent lung cancer induced by vinyl carbamate in $\mathrm{A} / \mathrm{J}$ mice. Cancer Res, 67, 2414-2419. 
35. Wang,X.J., Hayes,J.D., and Wolf,C.R. (2006) Generation of a stable antioxidant response element-driven reporter gene cell line and its use to show redox-dependent activation of nrf 2 by cancer chemotherapeutic agents. Cancer Res., 66, 10983-10994.

36. Thimmulappa,R.K., Scollick,C., Traore,K., Yates,M., Trush,M.A., Liby,K.T., Sporn,M.B., Yamamoto,M., Kensler,T.W., and Biswal,S. (2006) Nrf2-dependent protection from LPS induced inflammatory response and mortality by CDDO-Imidazolide. Biochem.Biophys.Res.Commun., 351, 883-889.

37. Li,B., Abdalrahman,A., Lai,Y., Janicki,J.S., Ward,K.W., Meyer,C.J., Wang,X.L., Tang,D., and Cui,T. (2014) Dihydro-CDDO-trifluoroethyl amide suppresses inflammatory responses in macrophages via activation of Nrf2. Biochem.Biophys.Res Commun., 444, 555-561.

38. Ahmad,R., Raina,D., Meyer,C., Kharbanda,S., and Kufe,D. (2006) Triterpenoid CDDO-Me Blocks the NF-\{kappa\}B Pathway by Direct Inhibition of IKKbeta on Cys-179. J.Biol.Chem., 281, 35764-35769.

39. Yore,M.M., Liby,K.T., Honda,T., Gribble,G.W., and Sporn,M.B. (2006) The synthetic triterpenoid 1-[2-cyano-3,12-dioxooleana-1,9(11)-dien-28-oyl]imidazole blocks nuclear factor\{kappa\}B activation through direct inhibition of I $\{$ kappa\}B kinase $\{$ beta\}. Mol.Cancer Ther., 5 , 3232-3239.

40. Stogios,P.J., Downs,G.S., Jauhal,J.J., Nandra,S.K., and Prive,G.G. (2005) Sequence and structural analysis of BTB domain proteins. Genome Biol., 6, R82.

41. Snyder,G.H., Cennerazzo,M.J., Karalis,A.J., and Field,D. (1981) Electrostatic influence of local cysteine environments on disulfide exchange kinetics. Biochemistry, 20, 6509-6519.

42. Thimmulappa,R.K., Lee,H., Rangasamy,T., Reddy,S.P., Yamamoto,M., Kensler,T.W., and Biswal,S. (2006) Nrf2 is a critical regulator of the innate immune response and survival during experimental sepsis. J.Clin.Invest., 116, 984-995.

43. Kobayashi,E.H., Suzuki,T., Funayama,R., Nagashima,T., Hayashi,M., Sekine,H., Tanaka,N., Moriguchi,T., Motohashi,H., Nakayama,K., and Yamamoto,M. (2016) Nrf2 suppresses macrophage inflammatory response by blocking proinflammatory cytokine transcription. Nat.Commun., 7, 11624.

44. Kobayashi,M., Li,L., Iwamoto,N., Nakajima-Takagi,Y., Kaneko,H., Nakayama,Y., Eguchi,M., Wada,Y., Kumagai,Y., and Yamamoto,M. (2008) The Antioxidant Defense System Keap1-Nrf2 Comprises a Multiple Sensing Mechanism for Responding to a Wide Range of Chemical Compounds. Mol.Cell Biol..

45. Gonzalez-Campo,A. and Amabilino,D.B. (2013) Biomolecules at interfaces: chiral, naturally. Top.Curr.Chem, 333, 109-156.

46. Gal,J. and Cintas,P. (2013) Early history of the recognition of molecular biochirality. Top.Curr.Chem, 333, 1-40.

47. Holland,R., Hawkins,A.E., Eggler,A.L., Mesecar,A.D., Fabris,D., and Fishbein,J.C. (2008) Prospective type 1 and type 2 disulfides of Keap1 protein. Chem.Res.Toxicol., 21, 2051-2060. 
48. McMahon,M., Lamont,D.J., Beattie,K.A., and Hayes,J.D. (2010) Keap1 perceives stress via three sensors for the endogenous signaling molecules nitric oxide, zinc, and alkenals. Proc.Natl.Acad.Sci.U.S.A, 107, 18838-18843.

49. Baird,L. and Dinkova-Kostova,A.T. (2013) Diffusion dynamics of the Keap1-Cullin3 interaction in single live cells. Biochem.Biophys.Res.Commun., 433, 58-65.

50. Baird,L., Lleres,D., Swift,S., and Dinkova-Kostova,A.T. (2013) Regulatory flexibility in the Nrf2-mediated stress response is conferred by conformational cycling of the Keap1-Nrf2 protein complex. Proc.Natl.Acad.Sci.U.S.A, 110, 15259-15264.

51. Linker,R.A., Lee,D.H., Ryan,S., van Dam,A.M., Conrad,R., Bista,P., Zeng,W., Hronowsky,X., Buko,A., Chollate,S., Ellrichmann,G., Bruck,W., Dawson,K., Goelz,S., Wiese,S., Scannevin,R.H., Lukashev,M., and Gold,R. (2011) Fumaric acid esters exert neuroprotective effects in neuroinflammation via activation of the Nrf2 antioxidant pathway. Brain, 134, 678-692.

52. Singh,J., Petter,R.C., Baillie,T.A., and Whitty,A. (2011) The resurgence of covalent drugs. Nat.Rev.Drug Discov., 10, 307-317.

53. Liu,Q., Sabnis,Y., Zhao,Z., Zhang,T., Buhrlage,S.J., Jones,L.H., and Gray,N.S. (2013) Developing irreversible inhibitors of the protein kinase cysteinome. Chem Biol., 20, 146-159.

54. Chin,M.P., Reisman,S.A., Bakris,G.L., O'Grady,M., Linde,P.G., McCullough,P.A., Packham,D., Vaziri,N.D., Ward,K.W., Warnock,D.G., and Meyer,C.J. (2014) Mechanisms contributing to adverse cardiovascular events in patients with type 2 diabetes mellitus and stage 4 chronic kidney disease treated with bardoxolone methyl. Am J Nephrol., 39, 499-508.

55. Chin,M.P., Wrolstad,D., Bakris,G.L., Chertow,G.M., de,Z.D., Goldsberry,A., Linde,P.G., McCullough,P.A., McMurray,J.J., Wittes,J., and Meyer,C.J. (2014) Risk factors for heart failure in patients with type 2 diabetes mellitus and stage 4 chronic kidney disease treated with bardoxolone methyl. J Card Fail., 20, 953-958.

56. de,Z.D., Akizawa,T., Audhya,P., Bakris,G.L., Chin,M., Christ-Schmidt,H., Goldsberry,A., Houser,M., Krauth,M., Lambers Heerspink,H.J., McMurray,J.J., Meyer,C.J., Parving,H.H., Remuzzi,G., Toto,R.D., Vaziri,N.D., Wanner,C., Wittes,J., Wrolstad,D., and Chertow,G.M. (2013) Bardoxolone methyl in type 2 diabetes and stage 4 chronic kidney disease. N.Engl.J Med., 369, 2492-2503. 